\title{
Fluid structure interaction problems: the necessity of a well posed, stable and accurate formulation
}

Jan Nordström and Sofia Eriksson

\section{Post Print}

N.B.: When citing this work, cite the original article.

Original Publication:

Jan Nordström and Sofia Eriksson, Fluid structure interaction problems: the necessity of a well posed, stable and accurate formulation, 2010, Communications in Computational Physics, (8), 1111-1138.

http://dx.doi.org/10.4208/cicp.260409.120210a

Copyright: Global Science Press

Postprint available at: Linköping University Electronic Press

http://urn.kb.se/resolve?urn=urn:nbn:se:liu:diva-68586 


\title{
Fluid Structure Interaction Problems: the Necessity of a Well Posed, Stable and Accurate Formulation
}

\author{
Jan Nordström ${ }^{1,2,3, *}$ and Sofia Eriksson ${ }^{3}$ \\ ${ }^{1}$ School of Mechanical, Industrial and Aeronautical Engineering, University of the \\ Witvatersrand, Johannesburg, WITS 2050, South Africa \\ 2 Department of Aeronautics and Systems Integration, FOI, The Swedish Defense \\ Research Agency, SE-164 90 Stockholm, Sweden \\ ${ }^{3}$ Department of Information Technology, Divison of Scientific Computing, Uppsala \\ University, SE-751 05 Uppsala, Sweden
}

\begin{abstract}
We investigate problems of fluid structure interaction type and aim for a formulation that leads to a well posed problem and a stable numerical procedure. Our first objective is to investigate if the generally accepted formulations of the fluid structure interaction problem are the only possible ones.

Our second objective is to derive a stable numerical coupling. To accomplish that we will use a weak coupling procedure and employ summation-by-parts operators and penalty terms. We compare the weak coupling with other common procedures. We also study the effect of high order accurate schemes.

In multiple dimensions this is a formidable task and we start by investigating the simplest possible model problem available. As a flow model we use the linearized Euler equations in one dimension and as the structure model we consider a spring.
\end{abstract}

AMS subject classifications: 65M12, 65M06,65M30,65M20,74F10,35Q35

Key words: fluid structure interaction, well posed, finite difference, high accuracy, stability

\section{Introduction}

Fluid structure interaction (FSI) problems occur in many different areas including aerodynamics ( [2], [4], [8]), acoustics ( [3], [7], [24]) and medicine ( [9], [12], [15], [14]). The specific FSI phenomenon called aerodynamic flutter is very dangerous and can cause accidents. It deserves attention in its own right, but it is also an example of a problem where most of the activity is due to the interaction between two separate physical problems.

Unless the coupling between these two problems is well posed and stable, either unphysical growth or damping can occur. (Some formulations try to avoid or decrease the

${ }^{*}$ Corresponding author. Email addresses: Jan. Nordstrom@it.uu. se (J. Nordström) 


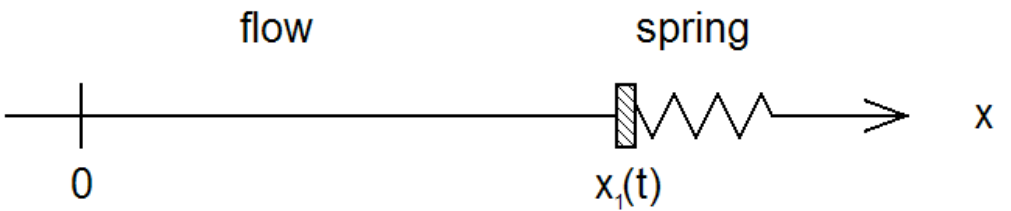

Figure 1: A one-dimensional flow problem interacting with a spring.

coupling problem, see for example [10]). The unphysical growth could result in expensive counter measures while the unphysical damping can provide a false sense of safety. We will investigate well-posedness and stability in detail and due to the complexity, we will start in one dimension and focus on the spatial problem.

First we investigate if the generally accepted formulation of the FSI problem is the only possible one (uniqueness) and such that it does not generate artificial energy growth. In short we will make sure that the coupling is well posed (see [11] for various concepts of well-posedness). Next, given that the problem is well posed, we will make sure that the numerical approximation is accurate and stable. We will study both the linear and non-linear problem.

The rest of the paper proceeds as follows. In section 2 we formulate the problem mathematically. Section 3 deals with the linear problem and well-posedness while the non-linear problem is discussed in section 4 . In section 5 we construct stable approximations for the linear semi-discrete approximation and describe the non-linear approximation. Section 6 presents the numerical experiments and conclusions are drawn in section 7.

\section{Mathematical formulation}

Our model problem is schematically depicted in Figure 1. The flow part to the left of $x_{1}(t)$ influence the structural part to the right and vice versa. The governing equations for the flow problem are the symmetric and linearized Euler equations in one dimension

$$
U_{t}+A U_{x}=0, \quad 0 \leq x \leq x_{1}(t)
$$

where

$$
A=\left[\begin{array}{ccc}
\bar{u} & \bar{c} / \sqrt{\gamma} & 0 \\
\bar{c} / \sqrt{\gamma} & \bar{u} & \bar{c} \sqrt{\frac{\gamma-1}{\gamma}} \\
0 & \bar{c} \sqrt{\frac{\gamma-1}{\gamma}} & \bar{u}
\end{array}\right], \quad U=\left[\begin{array}{c}
U^{1} \\
U^{2} \\
U^{3}
\end{array}\right]=\left[\begin{array}{c}
\bar{c}^{2} \rho / \sqrt{\gamma} \\
\bar{c} \bar{\rho} u \\
\bar{\rho} T / \sqrt{\gamma(\gamma-1) M_{\infty}^{4}}
\end{array}\right] .
$$

In (2.2), $\rho, u$ and $T$ represents the perturbation from the mean value of the density $(\bar{\rho})$, velocity $(\bar{u})$ and temperature $(\bar{T})$ respectively. Furthermore, $\bar{c}, M_{\infty}$ and $\gamma$ stands for the speed of sound at reference state, the Mach number at reference state and the ratio of 
specific heats. The equation of state is $p=\bar{c}^{2} \rho / \gamma+\bar{\rho} T /\left(\gamma M_{\infty}^{2}\right)$. For more details on how to obtain (2.1),(2.2), see [1], [19].

To simplify the analysis we transform equation (2.1) to a fixed domain using $\xi=x / x_{1}$ and $\tau=t$. We get

$$
x_{1}(\tau) U_{\tau}+\left(A-\xi \dot{x}_{1}\right) U_{\xi}=0 \quad 0 \leq \xi \leq 1 .
$$

The structural model, the spring equation, is given by

$$
m \ddot{\Delta x}+b \dot{\Delta x}+k \Delta x=f(U)
$$

where $\Delta x=x_{1}(t)-x_{E}, x_{E}=1$ being the equilibrium position of the spring. $m>0$ is the mass of the "surface" of the spring, $b \geq 0$ is the damping friction coefficient and $k>0$ is the spring constant. $f(U)$ is the net external force imposed by the flow. Let $V=\left[V_{1} V_{2}\right]^{T}=$ $\left[\begin{array}{ll}\Delta x & \dot{x}_{1}\end{array}\right]^{T}$ and rewrite (2.4) on first order system form as

$$
M V_{t}+K V=F(U)
$$

where

$$
M=\left[\begin{array}{cc}
k & 0 \\
0 & m
\end{array}\right], \quad K=\left[\begin{array}{cc}
0 & -k \\
k & b
\end{array}\right], \quad F=\left[\begin{array}{l}
0 \\
f
\end{array}\right]
$$

Equation (2.5) governs the structural part of the FSI problem.

Equations (2.3) and (2.5) must be coupled in order to describe the FSI problem correctly. One of the coupling terms is the forcing function $F(U)$ in (2.5). The other is the boundary condition for the flow equation (2.3) at $\xi=1$ which we formulate as

$$
B U=G V .
$$

The final form of the non-linear problem in terms of the dependent variables $U$ and $V$ (and changing notation from $\tau$ to $t$ ) are

$$
\begin{aligned}
\left(1+V_{1}\right) U_{t}+\left(A-\xi V_{2}\right) U_{\xi} & =0, \quad 0 \leq \xi \leq 1, \\
M V_{t}+K V & =F(U), \quad \xi=1 \\
B U & =G V . \quad \xi=1
\end{aligned}
$$

The problem (2.8) describes the coupling problem with the unknown forcing function $F(U)$ and coupling condition $B U=G V$. Boundary conditions at the left boundary and initial conditions must also be supplied. The linearized version of (2.8) is obtained by letting $V_{1}=V_{2}=0$ in the first equation.

We will consider both the linear and non-linear version of (2.8). The two versions differ at the right boundary where the coupling occurs but require the same treatment at the left boundary. 


\subsection{Well posed problems}

We will use the energy method as our main analysis tool and derive an equation for the energy-rate. We will give well posed boundary conditions at the left boundary but really focus on the interface coupling at the right boundary. At that boundary no external data is provided and for that reason, the following definition for well-posedness is appropriate.

Definition 2.1. The continuous problem (2.8) with homogeneous boundary conditions at the left boundary is well posed if a minimal number of boundary and coupling conditions are used and

$$
\frac{d}{d t}\|W\|_{2}^{2} \leq 0
$$

where $W=(U, V)^{T}$ and $\|W\|_{2}^{2}=\int_{0}^{1} W^{T} W d \xi$. The equality sign can only be valid if the damping in the structure problem is zero $(b=0)$.

Remark 2.1. Definition 2.1 does not allow for temporal solution growth, as would be necessary in order to bound arbitrary source terms, boundary data and non-linear terms. However, the only source of growth (both in the linear and non-linear case) in (2.8) is the coupling procedure at the interface. Our definition is therefore well suited for establishing well posedness of the coupling procedure, our primary goal. See [5] for a similar use of this definition.

Remark 2.2. Note that time integration of (2.9) leads to an estimate of the solution in terms of initial data and leads to the standard form of well posedness where the boundary data and forcing functions are assumed zero. See [11] for a discussion of various versions of well posedness.

Remark 2.3. The inequality (2.9) implies that the spatial operator in (2.8) is semi-bounded, see [11] for a discussion on semi-boundedness.

\subsection{Well posed boundary condition at the left boundary}

We use the energy method on the linear version of the first equation in (2.8) to arrive at the estimate

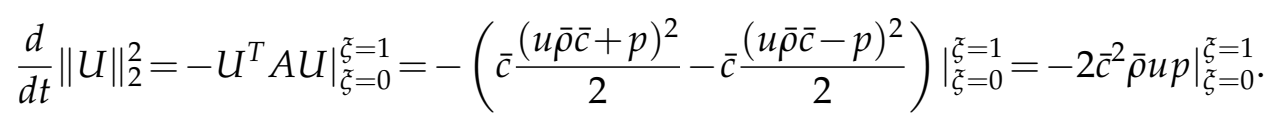

We have used $\|U\|_{2}^{2}=\int_{0}^{1} U^{T} U d \xi, \bar{u}=0$ (zero mean flow) and $p=\bar{c}^{2} \rho / \gamma+\bar{\rho} T /\left(\gamma M_{\infty}^{2}\right)$. Consider the left boundary at $\xi=0$. In general, a set of well posed boundary conditions are obtained by specifying the ingoing characteristic variable as a function of the outgoing variables and data on the boundary. In this case we use

$$
(u \bar{\rho} \bar{c}+p)=\alpha(u \bar{\rho} \bar{c}-p)+g(t) .
$$


which in the other variables become

$$
L U=g(t), \quad L=\left[(1+\alpha) \sqrt{\frac{1}{\gamma}},(1-\alpha),(1+\alpha) \sqrt{\frac{\gamma-1}{\gamma}}\right] .
$$

With $g(t)=0$ we obtain $\left.U^{T} A U\right|_{\xi=0}=\left(\alpha^{2}-1\right) \bar{c}(u \bar{\rho} \bar{c}-p)^{2} /\left.2\right|_{\xi=0}$ which implies that $-1 \leq$ $\alpha \leq 1$ to obtain a non-positive contribution in line with Definition 2.1. The most appealing choices of $\alpha$ is $0,-1$ and +1 , since that corresponds to giving boundary data to $u \bar{\rho} \bar{c}+p, u$ and $p$ respectively.

\section{The linear problem and well-posedness}

We will derive the coupling conditions at the right boundary for the linear problem in two distinctly different ways and start with the more conventional technique.

\subsection{Physical coupling approach at the right boundary}

The flow is adjacent to the spring which means that the velocity of the flow is equal to the velocity of the spring, i.e. $u(1, t)=\dot{x}_{1}$. The external force from the flow onto the spring is $a p_{1}$, where $a$ is the surface size (for simplicity we use $a=1$ ) and $p_{1}$ is the pressure at $\xi=1$. In summary the coupling conditions derived by physical reasoning are

$$
u(1, t)=\dot{x}_{1}, \quad f=p(1, t) .
$$

To make sure that the conditions in (3.1) are reasonable we must investigate well-posedness. If we multiply the second equation in (2.8) by $V^{T}$ we obtain

$$
\frac{d}{d t}\|V\|_{1}^{2}=2 V_{2} f-2 b V_{2}^{2}
$$

where $\|V\|_{1}^{2}=k V_{1}^{2}+m V_{2}^{2}$. A linear combination of the estimates (2.10) and (3.2), the coupling (3.1), and ignoring terms at $\xi=0$ leads to,

$$
\frac{d}{d t}\left(\|U\|_{2}^{2}+\delta\|V\|_{1}^{2}\right)=-\left.U^{T} A U\right|_{\xi=1}+\delta\left(2 V_{2} f-2 b V_{2}^{2}\right)=2 \dot{x}_{1} p_{1}\left(-2 \bar{c}^{2} \bar{\rho}+\delta\right)-2 \delta b \dot{x}_{1}^{2} .
$$

With $2 \bar{c}^{2} \bar{\rho}=\delta$ we cancel the indefinite term and arrive at a well posed coupling.

\subsection{Mathematical coupling approach at the right boundary}

In this section we will replace the specific physical coupling assumption (3.1) and only assume that the coupling conditions are linear relations, i.e.

$$
f(U)=\alpha^{T} U, \quad \alpha^{T}=\left[\alpha_{1}, \alpha_{2}, \alpha_{3}\right]
$$




$$
B U=\beta^{T} U=G V=\gamma^{T} V, \quad \beta^{T}=\left[\beta_{1}, \beta_{2}, \beta_{3}\right], \quad \gamma^{T}=\left[\gamma_{1}, \gamma_{2}\right] .
$$

Our objective is to see if we automatically arrive at the correct coupling conditions by demanding well-posedness.

The energy-method applied to the linear version of (2.8), ignoring terms at $\xi=0$ and a linear combination of the results lead to

$$
\frac{d}{d t}\left(\|U\|_{2}^{2}+\delta\|V\|_{1}^{2}\right)=-\left.U^{T} A U\right|_{\xi=1}+2 \delta\left(f V_{2}-b V_{2}^{2}\right) .
$$

The absence of $V_{1}$ implies that we can take $\gamma_{1}=0$ and $\gamma_{2}=1$. This means that we can solve for $V_{2}=\dot{x}_{1}$ in terms of $U$ and arrive at

$$
\frac{d}{d t}\left(\|U\|_{2}^{2}+\delta\|V\|_{1}^{2}\right)=-U^{T}\left[A-\delta\left(\beta \alpha^{T}+\alpha \beta^{T}\right)\right] U-2 \delta b U^{T} \beta \beta^{T} U
$$

For zero damping $(b=0)$ in the system, the symmetric matrix $\left[A-\delta\left(\beta \alpha^{T}+\alpha \beta^{T}\right)\right]$ must vanish for well-posedness. We have the condition

$$
\left[\begin{array}{ccc}
-2 \delta \alpha_{1} \beta_{1} & \bar{c} / \sqrt{\gamma}-\delta\left(\alpha_{1} \beta_{2}+\alpha_{2} \beta_{1}\right) & -\delta\left(\alpha_{1} \beta_{3}+\alpha_{3} \beta_{1}\right) \\
\bar{c} / \sqrt{\gamma}-\delta\left(\alpha_{1} \beta_{2}+\alpha_{2} \beta_{1}\right) & -2 \delta \alpha_{2} \beta_{2} & \bar{c} \sqrt{\frac{\gamma-1}{\gamma}}-\delta\left(\alpha_{2} \beta_{3}+\alpha_{3} \beta_{2}\right) \\
-\delta\left(\alpha_{1} \beta_{3}+\alpha_{3} \beta_{1}\right) & \bar{c} \sqrt{\frac{\gamma-1}{\gamma}}-\delta\left(\alpha_{2} \beta_{3}+\alpha_{3} \beta_{2}\right) & -2 \delta \alpha_{3} \beta_{3}
\end{array}\right]=0
$$

By inspection we find that the boundary matrix $\left[A-\delta\left(\beta \alpha^{T}+\alpha \beta^{T}\right)\right]$ vanish for

$$
\alpha_{2}=\beta_{1}=\beta_{3}=0, \quad \alpha_{1}=\frac{1}{\delta \beta_{2}} \frac{\bar{c}}{\sqrt{\gamma}}, \quad \alpha_{3}=\frac{1}{\delta \beta_{2}} \bar{c} \sqrt{\frac{\gamma-1}{\gamma}}
$$

or

$$
\beta_{2}=\alpha_{1}=\alpha_{3}=0, \quad \beta_{1}=\frac{1}{\delta \alpha_{2}} \frac{\bar{c}}{\sqrt{\gamma}}, \quad \beta_{3}=\frac{1}{\delta \alpha_{2}} \bar{c} \sqrt{\frac{\gamma-1}{\gamma}} .
$$

The relations in (3.8) and (3.9) lead to the following two alternative forms of coupling conditions

$$
\dot{x}_{1}=\left(\beta_{2} \bar{\rho} \bar{c}\right) u, \quad f=\left(\frac{\bar{c}}{\delta \beta_{2}}\right) p \quad \text { or } \quad \dot{x}_{1}=\left(\alpha_{2} \bar{\rho} \bar{c}\right) p, \quad f=\left(\frac{\bar{c}}{\delta \alpha_{2}}\right) u .
$$

The two relations in (3.10) are obtained without any physical knowledge at all.

To sort out the correct formulation we must add the requirement that the fluid is adjacent to the spring i.e. $u=\dot{x}_{1}$. That condition removes the second set of conditions in (3.9) and leads to the scaling $\beta_{2}=1 /(\bar{\rho} \bar{c})$. The choice $\delta=\bar{\rho} \bar{c}^{2}$ yield $f=p$.

Both the physical and mathematical coupling approach lead to the following formulation for the relations $f(U)$ and $B U=G V$ in terms of the matrices $\alpha^{T}, \beta^{T}$ and $\gamma^{T}$ as,

$$
f(U)=\alpha^{T} U, \quad \alpha^{T}=\left[\sqrt{\frac{1}{\gamma}}, 0, \sqrt{\frac{\gamma-1}{\gamma}}\right], \quad B=\beta^{T}=\left[0, \frac{1}{\bar{\rho} \bar{c}}, 0\right], \quad G=\gamma^{T}=[0,1] .
$$


We summarize the result so far for the linear problem (2.8) in the following proposition.

Proposition 3.1. The linearized version of (2.8) (obtained by letting $V_{1}=V_{2}=0$ in the first equation) combined with the boundary condition $(2.11),(2.12)$ and the coupling condition (3.1),(3.11) is well posed in the sense of Definition 2.1

Remark 3.1. To arrive at the correct coupling condition (3.1), (3.11) by only demanding well-posedness was not possible. That lead to two possible versions of coupling conditions in (3.9). The physical condition $u=\dot{x}_{1}$ (which can be seen as an accuracy requirement) had to be imposed in order to sort out the correct version.

Remark 3.2. The left boundary condition (2.11),(2.12) lead to a dissipative boundary treatment and a well posed problem in the sense of Definition 2.1. Other types of boundary conditions that do not lead to a norm decay are possible, and might lead to a well posed problem in some other sense.

\subsection{The continuous spectrum}

In the linear setting with $\bar{u}=0$ the system (2.1) have one zero eigenvalue and we can reduce our problem to a $2 \times 2$-system where $\rho$ and $T$ are linearly dependent. We get $\bar{c}^{2}(\gamma-1) M_{\infty}^{2} \rho-\bar{\rho} T=$ constant. To simplify further we will keep a linear combination of them, namely the pressure $p$. We get the reduced system

$$
W_{t}+\left(\begin{array}{cc}
0 & \bar{c} \\
\bar{c} & 0
\end{array}\right) W_{x}=0, \quad W=\left(\begin{array}{c}
u \bar{\rho} \bar{c} \\
p
\end{array}\right),
$$

which on diagonal form becomes

$$
w_{t}+\Lambda w_{x}=0, \quad w=\frac{1}{\sqrt{2}}\left[\begin{array}{l}
u \bar{\rho} \bar{c}-p \\
u \bar{\rho} \bar{c}+p
\end{array}\right], \quad \Lambda=\left[\begin{array}{rr}
-\bar{c} & 0 \\
0 & \bar{c}
\end{array}\right] .
$$

By Laplace transforming (3.13) we obtain $s \hat{w}+\Lambda \hat{w}_{x}=0$. The Ansatz: $\hat{w}=\Psi e^{\lambda x}$ leads to $(s I+\lambda \Lambda) \Psi=0$. A non-trivial solution yields $\lambda_{1,2}= \pm s / \bar{c}, \Psi_{1}=\left[\begin{array}{ll}1 & 0\end{array}\right]^{T}, \Psi_{2}=\left[\begin{array}{ll}0 & 1\end{array}\right]^{T}$ and finally

$$
\hat{w}=\sigma_{1} \Psi_{1} e^{\lambda_{1} x}+\sigma_{2} \Psi_{2} e^{\lambda_{2} x}=\left(\sigma_{1} e^{+s x / \bar{c}}, \sigma_{2} e^{-s x / \bar{c}}\right)^{T} .
$$

The coefficients $\sigma_{1}$ and $\sigma_{2}$ will be determined by the boundary conditions.

At the left boundary we consider the boundary condition (2.11) with $\alpha=+1,-1,0$ respectively. That leads to

$$
\begin{aligned}
& \left.\sqrt{2} \hat{p}\right|_{0}=\left.\hat{w}_{2}\right|_{0}-\left.\hat{w}_{1}\right|_{0} \quad \quad=\sigma_{2}-\sigma_{1} \quad=\hat{g}_{1} \\
& \left.\sqrt{2} \bar{\rho} \bar{c} \hat{u}\right|_{0}=\left.\hat{w}_{2}\right|_{0}+\left.\hat{w}_{1}\right|_{0} \quad=\sigma_{2}+\sigma_{1} \quad=\hat{g}_{2} \\
& \left(\left.\bar{\rho} \bar{c} \hat{u}\right|_{0}+\left.\hat{p}\right|_{0}\right) / \sqrt{2}=\left.\hat{w}_{2}\right|_{0} \quad=\sigma_{2} \quad=\hat{g}_{3}
\end{aligned}
$$


At the right boundary we specify the velocity (required for well-posedness), hence

$$
\left.\sqrt{2} \bar{\rho} \bar{c} \hat{u}\right|_{1}=\left.\hat{w}_{2}\right|_{1}+\left.\hat{w}_{1}\right|_{1}=\sigma_{2} e^{-s / \bar{c}}+\sigma_{1} e^{s / \bar{c}}=\hat{g}_{4}=\sqrt{2} \bar{\rho} \bar{c} \hat{V}_{2}
$$

The data $g_{4}$ is obtained from the the structural part which we consider next.

The Laplace transform of (2.5) yields

$$
s \hat{V}+\left[\begin{array}{cc}
0 & -1 \\
k / m & b / m
\end{array}\right] \hat{V}=\left[\begin{array}{c}
0 \\
\left.\hat{p}\right|_{1} / m
\end{array}\right] \Rightarrow \hat{V}=\frac{\left.\hat{p}\right|_{1}}{m s^{2}+b s+k}\left[\begin{array}{l}
1 \\
s
\end{array}\right] .
$$

The boundary conditions (3.18) now yields

$$
\frac{s\left(\left.\hat{w}_{2}\right|_{1}-\left.\hat{w}_{1}\right|_{1}\right)}{\sqrt{2}\left(m s^{2}+b s+k\right)}=\left.\frac{\left.\hat{w}_{2}\right|_{1}+\left.\hat{w}_{1}\right|_{1}}{\sqrt{2} \bar{\rho} \bar{c}} \Rightarrow \hat{w}_{1}\right|_{1}(1+\varphi)+\left.\hat{w}_{2}\right|_{1}(1-\varphi)=0,
$$

where $\varphi(s)=\bar{\rho} \bar{c} s /\left(m s^{2}+b s+k\right)$ and $\left.\hat{w}_{1}\right|_{1}=\sigma_{1} e^{s} / \bar{c}$ and $\left.\hat{w}_{2}\right|_{1}=\sigma_{2} e^{-s / \bar{c}}$.

The linear equations system for determining the coefficients $\sigma_{1}$ and $\sigma_{2}$ can formally be written

$$
C(s) \sigma=\hat{g}
$$

where

$$
C(s)=\left[\begin{array}{cc}
-\alpha & 1 \\
(1+\varphi) e^{s / \bar{c}} & (1-\varphi) e^{-s / \bar{c}}
\end{array}\right], \quad \sigma=\left[\begin{array}{c}
\sigma_{1} \\
\sigma_{2}
\end{array}\right], \quad g=\left[\begin{array}{c}
\hat{g}_{i} \\
0
\end{array}\right] .
$$

A unique solution is obtained for

$$
|C(s)|=-\left(e^{s / \bar{c}}(1+\varphi)+e^{-s / \bar{c}} \alpha(1-\varphi)\right) \neq 0 .
$$

The $s$ values that lead to a singular matrix $C(s)$ gives the spectrum or the eigenvalues of the problem. The value of $\alpha=1$ corresponds to giving data to $p, \alpha=-1$ to $u$ and $\alpha=0$ to $\bar{\rho} \bar{c} u+p$. If the damping of the system is zero $(b=0)$ and data is given to either $u$ or $p$, then all eigenvalues will be on the imaginary axis. Each eigenvalue correspond to an eigenfrequency of the coupled system.

\section{The non-linear problem and well-posedness}

The full non-linear representation of the flow system is given by (2.3) or equivalently the first equation in (2.8). The energy method gives

$$
\frac{d}{d t}\left(\left(1+V_{1}\right)\|U\|_{2}^{2}\right)=-\left.U^{T}\left(A-V_{2} I_{3}\right) U\right|_{\xi=1}+\left.U^{T} A U\right|_{\xi=0} .
$$

In comparison with the linear case, the norm is slightly modified and at the interface between the fluid and the spring, an additional term $\left.V_{2} U^{T} U\right|_{\xi=1}$ is added on. The conditions at the left boundary is the same as in the linear case. The term $\left.V_{2} U^{T} U\right|_{\xi=1}$ is indefinite since $V_{2}$ changes sign. This means that the non-linear problem is not well-posed in the sense of Definition 2.1. We summarize this as follows. 
Proposition 4.1. The non-linear problem (2.8) combined with the boundary condition $(2.11),(2.12)$ and the coupling condition (3.1),(3.11) is not well posed in the sense of Definition 2.1.

Remark 4.1. Note that we do not claim that the non-linear problem (2.8) is ill-posed. It does not satisfy the requirements in Definition 2.1 and hence it is not well posed in that sense. However, it might be well posed in some other sense.

\section{The semi-discrete approximation and stability}

We will do numerical calculations using both the linear and the non-linear equations. For both cases we will use the linear coupling treatment developed above. With a slight abuse of notation we use $U, V$ both for the continuous and discrete vectors.

\subsection{The semi-discrete linear formulation}

The discrete version of the linearized flow equation in (2.8) with the boundary and interface conditions (2.11),(2.12) (3.1),(3.11) imposed as penalty terms is written as

$$
U_{t}+\left(P^{-1} Q \otimes A\right) U=\left(P^{-1} E_{0} \otimes \Pi\right)\left(U-G_{0}\right)+\left(P^{-1} E_{N} \otimes \Sigma\right)\left(U-G_{N}\right) .
$$

The grid in (5.1) consist of $N+1$ equidistant grid points and the solution is organized as $U=\left(U_{0}, U_{1}, . ., U_{i}, . ., U_{N-1}, U_{N}\right)^{T}$ with $U_{i}=\left(U^{1}, U^{2}, U^{3}\right)_{i}$.

We have used a summation-by-parts (SBP) difference operator $P^{-1} Q$ (see [13], [23]) and imposed the boundary conditions weakly using the SAT technique [6]. The SBP difference operators satisfy the relations

$$
P=P^{T}>0, \quad Q+Q^{T}=E_{N}-E_{0}=\operatorname{diag}(-1,0, \ldots 0,1),
$$

and hence they mimic integration by parts perfectly. More details on the weak imposition of boundary and interface conditions using the SAT technique will be given below. For a read-up on this technique see [6], [17], [21], [22].

The left penalty term on the right-hand side of (5.1) is connected to the left continuous boundary condition $(L U=g)$ by the following relations.

$$
\left(P^{-1} E_{0} \otimes \Pi\right)\left(U-G_{0}\right)=\left(P^{-1} e_{0} \otimes \tau^{0}\right)\left(e_{0}^{T} \otimes L\right)\left(U-G_{0}\right)=\left(P^{-1} e_{0} \otimes \tau^{0}\right)\left(L U_{0}-g\right) .
$$

The boundary condition at the utmost right in (5.3) is defined in $(2.12), e_{0}=(1,0, . .0,0)^{T}$, $e_{0} e_{0}^{T}=E_{0}, \Pi=\tau^{0} L, G_{0}=\left(g^{0}, 0, \ldots, 0\right)^{T}, g^{0}=\left(g_{0}, g_{1}, g_{2}\right)^{T}$ and $g=L g^{0}$. The penalty matrix $\Pi$ has an undefined component $\tau^{0}=\left(\tau_{1}^{0}, \tau_{2}^{0}, \tau_{3}^{0}\right)$ which will be determined by stability requirements.

The right penalty term on the right-hand side of (5.1) is connected to the right continuous interface condition $(B U=G V)$ by the following relations.

$$
\left(P^{-1} E_{N} \otimes \Sigma\right)\left(U-G_{N}\right)=\left(P^{-1} e_{N} \otimes \tau^{N}\right)\left(e_{N}^{T} \otimes B\right)\left(U-G_{N}\right)=\left(P^{-1} e_{N} \otimes \tau^{N}\right)\left(B U_{N}-G V\right) .
$$


The interface condition at the utmost right in (5.4) is defined in $(3.11), e_{N}=(0,0, . .0,1)^{T}$, $e_{N} e_{N}^{T}=E_{N}, \Sigma=\tau^{N} B, G_{N}=\left(0,0, \ldots, 0, g^{N}\right)^{T}, G V=B g^{N}=V_{2}$ and $g^{N}=\left(U_{N}^{1}, \bar{\rho} \bar{c} V_{2}, U_{N}^{3}\right)^{T}$. The penalty matrix $\Sigma$ has an undefined component $\tau^{N}=\left(\tau_{1}^{N}, \tau_{2}^{N}, \tau_{3}^{N}\right)$ which will be determined by stability requirements.

By multiplying (5.1) with $U^{T}\left(P \otimes I_{3}\right)$ from the left, adding the transpose and using the relation (5.2) we obtain

$$
\frac{d}{d t}\|U\|_{\bar{P}}^{2}+U^{T}\left(\left(-E_{0}+E_{N}\right) \otimes A\right) U=2 U_{0}^{T} \Pi\left(U_{0}-g^{0}\right)+2 U_{N}^{T} \Sigma\left(U_{N}-g^{N}\right),
$$

where $\|U\|_{\bar{P}}^{2}=U^{T} \bar{P} U$. For convenience we have introduced the notation $\bar{P}=P \otimes I_{3}$. By adding the estimate for the structure part using the same scaling $\delta$ as in the continuous case we find

$$
\begin{aligned}
\frac{d}{d t}\left(\|U\|_{\bar{P}}^{2}+\delta\|V\|_{1}^{2}\right) & =U_{0}^{T} A U_{0}+2 U_{0}^{T} \Pi\left(U_{0}-g^{0}\right) \\
& -U_{N}^{T} A U_{N}+2 U_{N}^{T} \Sigma\left(U_{N}-g^{N}\right)+2 \delta f V_{2}-2 \delta b V_{2}^{2}
\end{aligned}
$$

\subsection{The semi-discrete non-linear formulation}

In the non-linear discrete case we have an additional matrix operator $\Xi$ which models the impact of the coordinate $\xi$. Since the matrices $Q$ and $\Xi$ do not commute we must split the derivative terms in a symmetric and skew-symmetric part, see [20] for more details. Consider the first equation in (2.8). We split the term as

$$
\xi U_{\xi}=\left(\xi U_{\xi}\right) / 2+\left((\xi U)_{\xi}-U\right) / 2,
$$

and use the following discrete representations

$$
A U_{\xi} \sim\left(P^{-1} Q \otimes A\right) U, \quad \xi U_{\xi} \sim\left(\Xi P^{-1} Q \otimes I_{3}\right) U, \quad(\xi U)_{\xi} \sim\left(P^{-1} Q \Xi \otimes I_{3}\right) U .
$$

We have used $\xi \sim \Xi=\operatorname{diag}(01 / N 2 / N \cdots 1)$. The semi-discrete version of the non-linear flow equation in (2.8) is written as

$$
\begin{aligned}
\left(1+V_{1}\right) U_{t} & +\left(P^{-1} Q \otimes A\right) U-V_{2}\left(\left(\Xi P^{-1} Q \otimes I_{3}\right)+\left(P^{-1} Q \Xi \otimes I_{3}\right)-\left(I_{N} \otimes I_{3}\right)\right) U / 2 \\
& =\left(P^{-1} E_{0} \otimes \Pi\right)\left(U-G_{0}\right)+\left(P^{-1} E_{N} \otimes \Sigma\right)\left(U-G_{N}\right) .
\end{aligned}
$$

The energy rate is given by multiplying (5.8) from the left by $U^{T}\left(P \otimes I_{3}\right)$, adding the transpose, using (5.2), adding the spring estimate and observing that $\left(1+V_{1}\right)_{t}=V_{2}$. We have

$$
\begin{aligned}
\frac{d}{d t}\left(\left(1+V_{1}\right)\|U\|_{\bar{P}}^{2}+\delta\|V\|_{1}^{2}\right) & =U_{0}^{T} A U_{0}+2 U_{0}^{T} \Pi\left(U_{0}-g^{0}\right) \\
& -U_{N}^{T}\left(A-V_{2} I_{3}\right) U_{N}+2 U_{N}^{T} \Sigma\left(U_{N}-g^{N}\right)+2 \delta f V_{2}-2 \delta b V_{2}^{2}
\end{aligned}
$$

The difference between the linear (5.6) and non-linear (5.9) estimates is the form of the norm and the additional term $V_{2} U_{N}^{T} U_{N}$ (the same difference as in the continuous case). 


\subsection{Stability}

We will define stability in a similar manner as we defined well posedness for the continuous problem. We focus on the interface coupling at the right boundary (even though the imposition of the boundary conditions at the left boundary are also considered). At that interface no external data is provided and for that reason, the following definition of stability is appropriate.

Definition 5.1. Consider the linear (5.1) and non-linear (5.8) numerical approximations of the problem (2.8) with homogeneous boundary conditions at the left boundary. These approximations are stable if

$$
\frac{d}{d t}\|W\|_{3}^{2} \leq 0
$$

where $W=(U, V)^{T}$. In the linear case, see (5.6), we have the norm $\|W\|_{3}^{2}=\|U\|_{\bar{P}}^{2}+\delta\|V\|_{1}^{2}$. The norm in the non-linear case, see (5.9), is $\|W\|_{3}^{2}=\left(1+V_{1}\right)\|U\|_{\bar{P}}^{2}+\delta\|V\|_{1}^{2}$.

Remark 5.1. Just as in the continuous case (see Remark 2.1), Definition 5.1 does not allow for a general temporal solution growth but is well suited for establishing stability of the coupling procedure. See [5] for a similar use of this definition.

Remark 5.2. Time integration of (5.10) leads to an estimate of the solution in terms of initial data and the standard form of stability. See Remark 2.2 and [11] for a discussion of various stability concepts.

Remark 5.3. The inequality (5.10) implies that the spatial operator in (2.8) is semi-bounded, see Remark 2.3 and [11] for a discussion on semi-boundedness.

\subsection{A stable left boundary procedure}

Consider the boundary terms (BT) of (5.6) at $\xi=0$ and the boundary condition (2.11),(2.12). By assuming that $g(t)=0$ we obtain,

$$
B T=U^{T}\left(A+\Pi+\Pi^{T}\right) U, \quad \Pi=\tau^{0} L=\left[\begin{array}{ccc}
\tau_{1}^{0} L_{1} & \tau_{1}^{0} L_{2} & \tau_{1}^{0} L_{3} \\
\tau_{2}^{0} L_{1} & \tau_{2}^{0} L_{2} & \tau_{2}^{0} L_{3} \\
\tau_{3}^{0} L_{1} & \tau_{3}^{0} L_{2} & \tau_{3}^{0} L_{3}
\end{array}\right] .
$$

Recall that the boundary operator $L=\left(L_{1}, L_{2}, L_{3}\right)$ is a function of the parameter $\alpha$, see (2.12), which lead to well posedness if $-1 \leq \alpha \leq+1$. Recall also that $\alpha=-1,0,+1$ corresponds to giving boundary data to $u \bar{\rho} \bar{c}+p, u$ and $p$ respectively.

The specific choices of $\alpha$ together with the requirement that $B T$ must be negative semi-definite lead to the following three penalty coefficients,

$$
\tau_{u}^{0}=\frac{\bar{c}}{2}\left[\begin{array}{c}
-\frac{1}{\sqrt{\gamma}} \\
\tau \\
-\sqrt{\frac{\gamma-1}{\gamma}}
\end{array}\right], \quad \tau_{p}^{0}=\frac{\bar{c}}{2}\left[\begin{array}{c}
\tau \frac{1}{\sqrt{\gamma}} \\
-1 \\
\tau \sqrt{\frac{\gamma-1}{\gamma}}
\end{array}\right], \quad \tau_{\bar{\rho} \bar{c} u+p}^{0}=\tau \bar{c}\left[\begin{array}{c}
\frac{1}{\sqrt{\gamma}} \\
1 \\
\sqrt{\frac{\gamma-1}{\gamma}}
\end{array}\right] .
$$


For stability reasons, the semi-bounded parameter $\tau$ in (5.11) has to be negative semidefinite for $\tau_{u}^{0}, \tau_{p}^{0}$ and less than $-1 / 4$ for $\tau_{\bar{\rho} \bar{c} u+p}^{0}$.

The penalty coefficients in (5.11) above lead to the following three penalty matrices,

$$
\begin{aligned}
\Pi_{u} & =\bar{c}\left[\begin{array}{ccc}
0 & -\frac{1}{\sqrt{\gamma}} & 0 \\
0 & \tau & 0 \\
0 & -\sqrt{\frac{\gamma-1}{\gamma}} & 0
\end{array}\right], \quad \Pi_{p}=\bar{c}\left[\begin{array}{ccc}
\tau \frac{1}{\gamma} & 0 & \tau \frac{\sqrt{\gamma-1}}{\gamma} \\
-\frac{1}{\sqrt{\gamma}} & 0 & -\sqrt{\frac{\gamma-1}{\gamma}} \\
\tau \frac{\sqrt{\gamma-1}}{\gamma} & 0 & \tau \frac{\gamma-1}{\gamma}
\end{array}\right], \quad \tau \leq 0 \\
\Pi_{\bar{\rho} \bar{c} u+p} & =\tau \bar{c}\left[\begin{array}{ccc}
\frac{1}{\gamma} & \frac{1}{\sqrt{\gamma}} & \frac{\sqrt{\gamma-1}}{\gamma} \\
\frac{1}{\sqrt{\gamma}} & 1 & \sqrt{\frac{\gamma-1}{\gamma}} \\
\frac{\sqrt{\gamma-1}}{\gamma} & \sqrt{\frac{\gamma-1}{\gamma}} & \frac{\gamma-1}{\gamma}
\end{array}\right], \quad \tau \leq-\frac{1}{4} .
\end{aligned}
$$

The penalty matrices in (5.12) and (5.13) lead to a stable left boundary treatment for both the linear and non-linear case. The choice of $\tau$ on the stability borders ( 0 for $(5.12)$ and $-1 / 4$ for (5.13)) give a minimal numerical damping.

\subsection{A stable right boundary procedure for the linear case}

Consider the coupling terms $(C T)$ at the right boundary where $\xi=1$. We have

$$
C T=-U_{N}^{T} A U_{N}+2 U_{N}^{T} \Sigma\left(U_{N}-g^{N}\right)+2 \bar{\rho} \bar{c}^{2} f V_{2}-2 \bar{\rho} \bar{c}^{2} b V_{2}^{2} .
$$

The coupling conditions $f=p_{N}, g^{N}=\left(U_{N}^{1}, \bar{\rho} \bar{c} V_{2}, U_{N}^{3}\right)^{T}, \Sigma=\tau^{N} B$ and zero damping $(b=0)$ leads to

$$
\begin{aligned}
C T & =-2 \bar{\rho} \bar{c}^{2} u_{N} p_{N}+2 U_{N}^{T}\left[\begin{array}{ccc}
0 & \tau_{1}^{N} / \bar{\rho} \bar{c} & 0 \\
0 & \tau_{2}^{N} / \bar{\rho} \bar{c} & 0 \\
0 & \tau_{3}^{N} / \bar{\rho} \bar{c} & 0
\end{array}\right]\left[\begin{array}{c}
0 \\
\left(U^{2}\right)_{N}-\bar{\rho} \bar{c} V_{2} \\
0
\end{array}\right]+2 \bar{\rho} \bar{c}^{2} V_{2} p_{N} \\
& =2\left(u_{N}-V_{2}\right)\left(-\bar{\rho} \bar{c}^{2} p_{N}+U_{N}^{T} \tau^{N}\right) .
\end{aligned}
$$

Clearly, $\tau^{N}=\bar{\rho} \bar{c}^{2}(1 / \sqrt{\gamma}, 0, \sqrt{(\gamma-1) / \gamma})^{T}$ leads to $C T=0$ and a stable boundary treatment for the right boundary. The final form of $\Sigma$ is

$$
\Sigma=\left[\begin{array}{ccc}
0 & \bar{c} / \sqrt{\gamma} & 0 \\
0 & 0 & 0 \\
0 & \bar{c} \sqrt{\frac{\gamma-1}{\gamma}} & 0
\end{array}\right] .
$$

Remark 5.4. Note the similarity between the matrix elements in the penalty matrices (5.12),(5.13),(5.15) and the matrix elements of $A$ in (2.2)

Just as in the continuous case (see Theorem 3.1), we summarize the result so far for the linear approximation in the following proposition. 
Proposition 5.1. The semi-discrete approximation (5.1) of the linear version of (2.8) is stable in the sense of Definition 5.1 if the penalty matrices (5.12),(5.13) and (5.15) are used to impose the boundary condition $(2.11),(2.12)$ and the coupling condition $(3.1),(3.11)$, respectively.

Remark 5.5. Just as in the continuous case, see Remark 3.2, other types of less restrictive stability definitions are of course possible.

\subsection{The right boundary procedure in the non-linear case}

By applying the exact same procedure (the penalty matrix in (5.15) with the same data) for the non-linear case we obtain

$$
C T=V_{2} U_{N}^{T} U_{N}
$$

Clearly this cannot be shown to be stable since the sign of the right-hand-side varies. We vill investigate numerically what this means and come back to this issue.

We summarize the result so far as follows.

Proposition 5.2. The semi-discrete approximation (5.8) of the non-linear version of (2.8) together with the the penalty matrices (5.12),(5.13) and (5.15) is not stable in the sense of Definition 5.1.

Remark 5.6. Note that we do not claim that the approximation (5.8) of the non-linear problem (2.8) is unstable. It does not satisfy the requirements in Definition 5.1 and hence it is not stable in that sense. However, it might be stable in some other sense.

\section{Numerical simulations}

The semi-discrete FSI problem was integrated in time as a large system of ordinary differential equation of the form $y_{t}=M(t) y+g(t)$, where $y=[U ; V]$ and $g(t)$ includes the boundary data. To integrate the system in time we used the classical 4th order RungeKutta method. In the linear case, $M$ is a constant $(3 N+5) \times(3 N+5)$ matrix. In the nonlinear case, $M=M(t)$ is slightly modified. The structure of the matrix $M$ depend on the boundary and coupling conditions. For details, see Appendix A. In all the calculations below we have used the parameters $m=0.1, b=0, k=1, \bar{\rho}=\bar{c}=1, \bar{u}=0, \gamma=1.4$.

\subsection{Verification of the numerical approximation}

We start by verifying that our numerical approximation (5.1) together with the second equation in (2.8) is correct by checking it using the method of manufactured solutions. We construct our manufactured solution such that the interface relation $U^{2}=\bar{\rho} \bar{c} V_{2}$ or equivalently, $u(1, t)=\dot{x}_{1}(t)$ is satisfied. Next we insert forcing functions that balance 


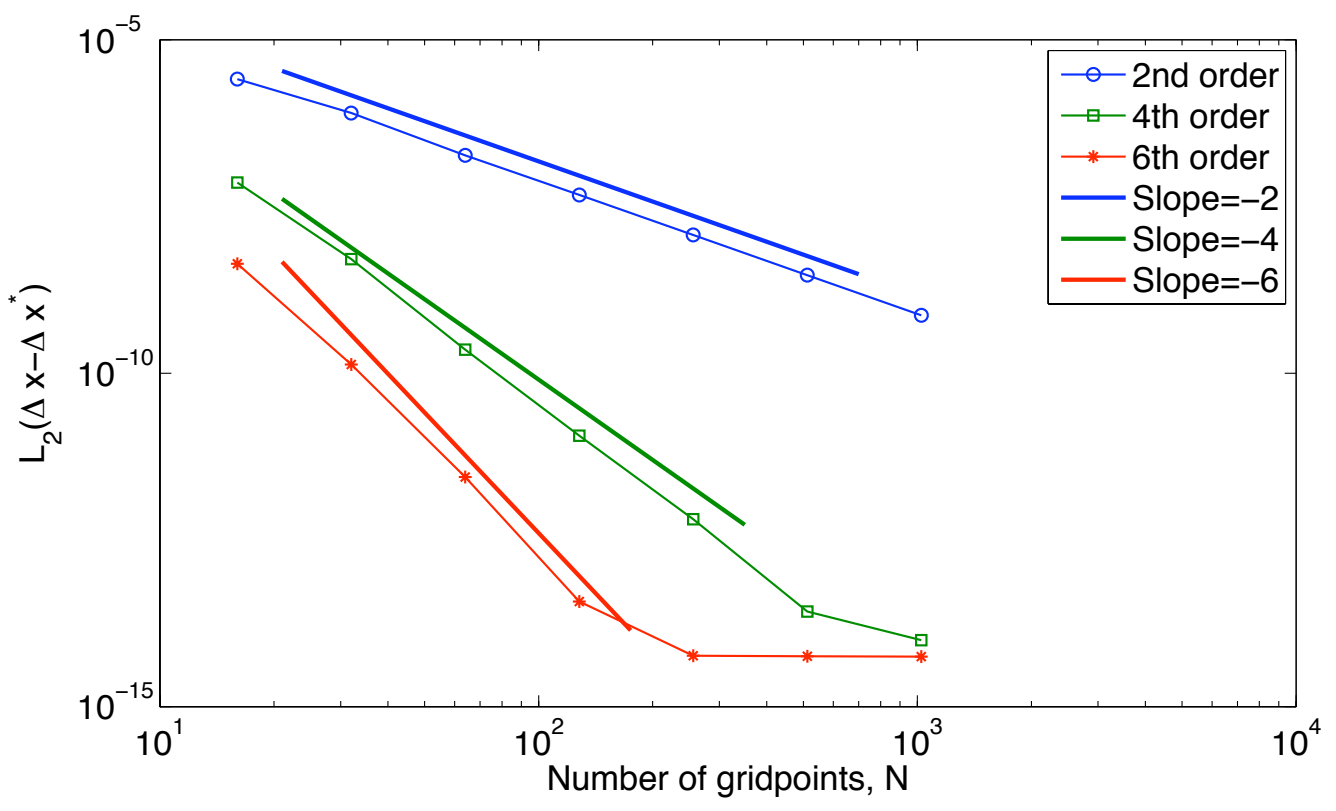

Figure 2: Convergence rates obtained using the method of manufactured solutions.

the residuals from the manufactured solutions on the right-hand-side in the equations. Finally we compute the $L_{2}$ error in time for the position $V_{1}=\Delta x(t)$, and plot it against the number of grid points $N$ in space. The $L_{2}$ error is computed on a grid with $n$ time levels. As can be seen in Figure 2, the results converge with the correct orders of accuracy.

We also verify our numerical approximation by making sure that the numerical eigenvalues (or eigenfrequencies) converge to the spectrum of the continuous operator. With the damping coefficient of the spring $b$ being zero and specifying the pressure $p$ at the left boundary, the continuous spectra is purely imaginary.

In Figure 3, the eigenvalues of the linear discrete (second order accurate) operator $M$ are shown together with the continuous spectra. The position of the continuous eigenvalues $s$ are marked as dashed lines. The numerical eigenvalues converge to the continuous ones as the number of grid points increases (plus a number of additional purely numerical eigenvalues). The eigenvalues are purely imaginary and symmetrically distributed around the real axis (we use the penalty matrix in (5.12) for the pressure $p$ with zero numerical damping $(\tau=0)$ ). Only the ones with positive imaginary part are shown.

The eigenvectors corresponding to the first four eigenvalues for $N=16$ are shown in Figure 4. Note that the eigenvectors related to the continuous spectrum are smooth while those related to the purely numerical (spurious) eigenvalues are highly oscillatory.

In Figures 5 and 6 we show the difference between the second, fourth and sixth order accurate schemes when computing eigenfrequencies. The higher order ones are more accurate and converges faster. In Figure 6 we have only kept the numerical eigenvalues 


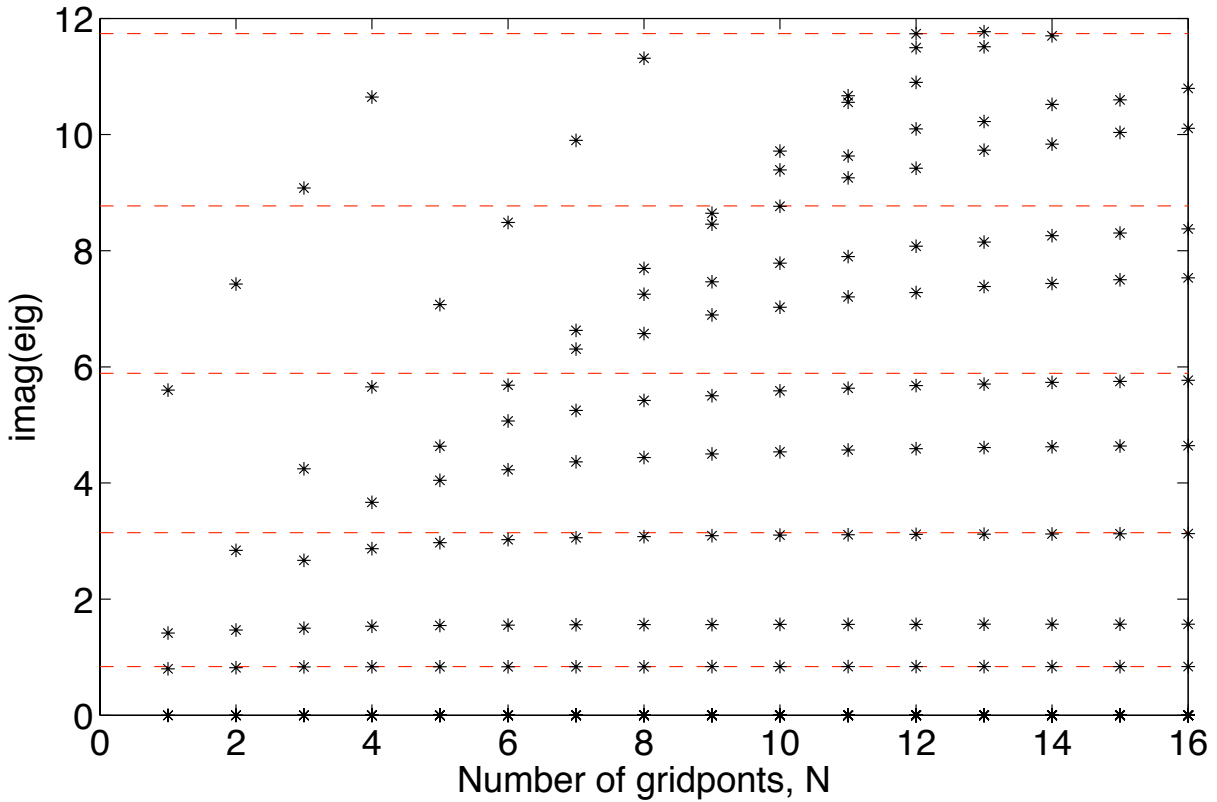

Figure 3: Numerical eigenvalues of the operator $M$ as a function of the number of grid-points. The dashed lines indicate the position of the continuous eigenvalues $s$.

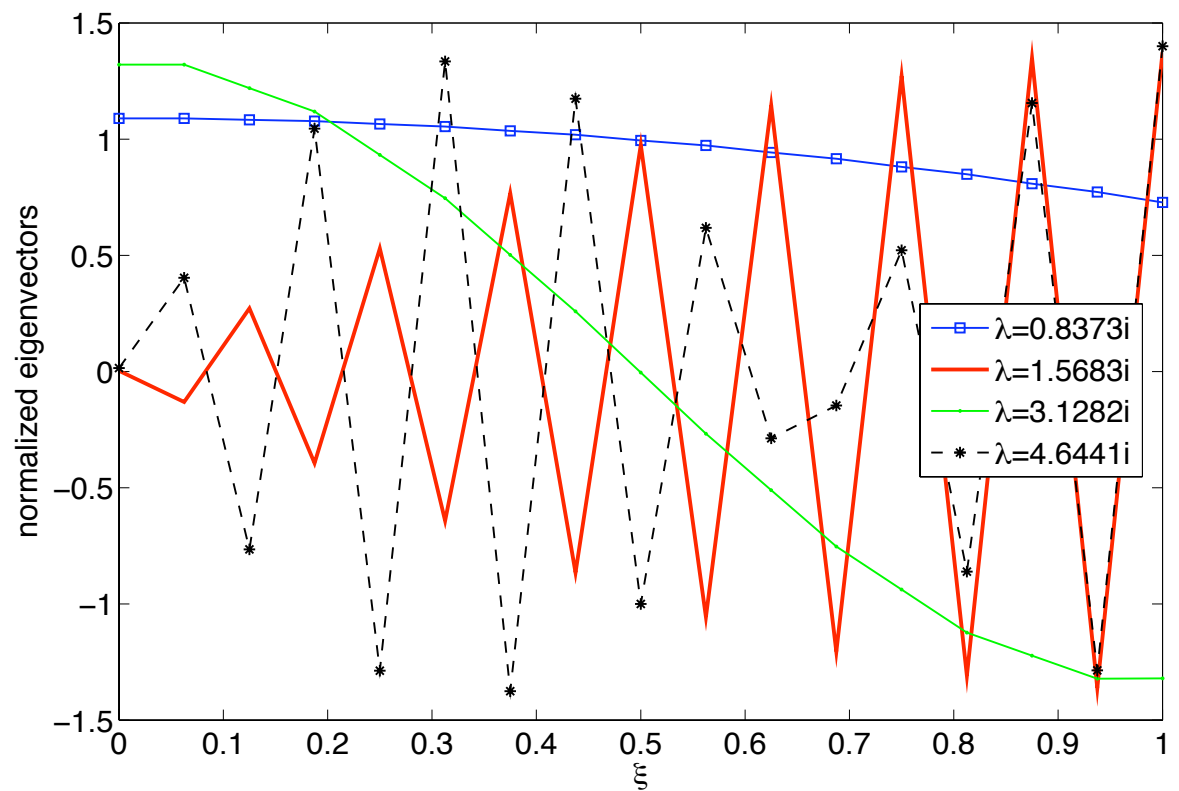

Figure 4: Eigenvectors related to the first four eigenvalues for $N=16$. The two smooth eigenvectors are related to the continuous spectrum. The two oscillatory eigenvectors are related to numerical or spurious eigenvalues. 


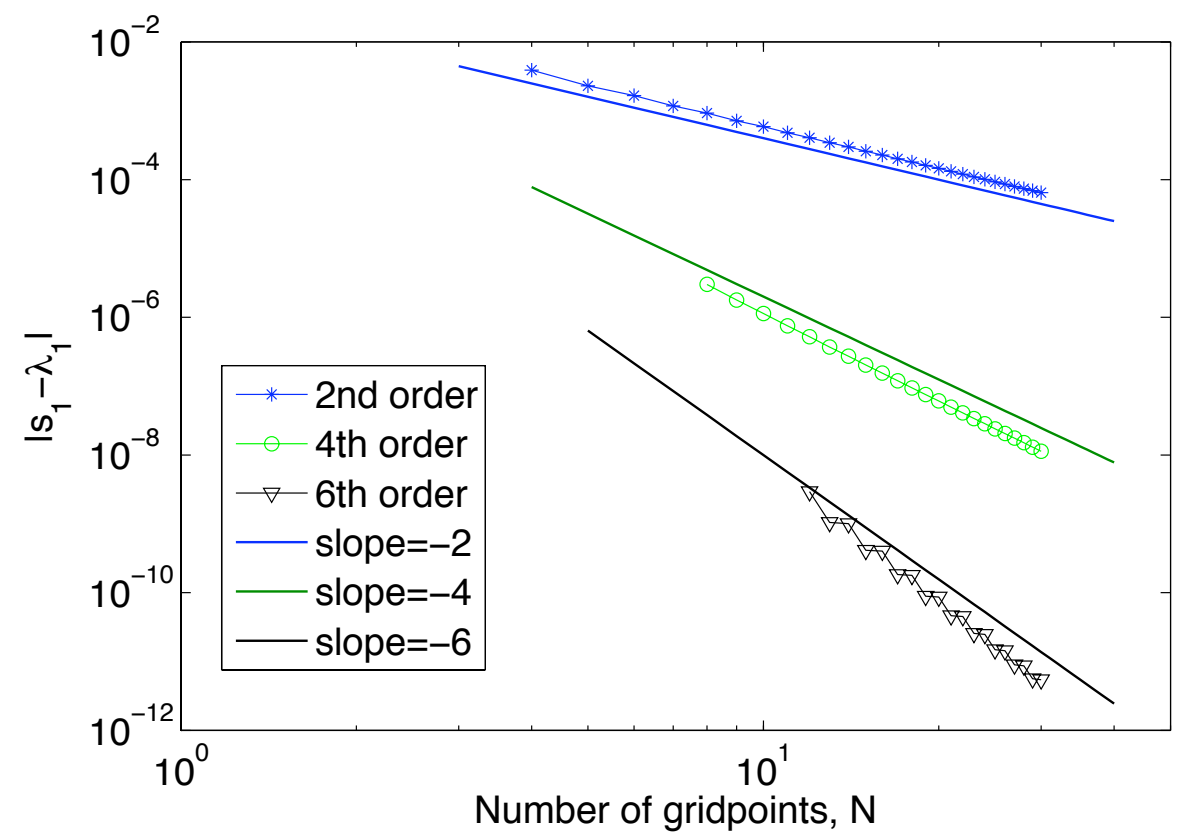

Figure 5: The convergence of the lowest numerical eigenfrequency $(\omega=0.83756479484021)$ for different orders of accuracy. Weak imposition of boundary conditions is used.

that converge to the real eigenvalues. The purely numerical ones are removed for clarity, cf Figure 3 where all the eigenvalues are shown.

To further check our numerical scheme we gave the spring a small displacement $\left(V_{1}(0)=\Delta x(0)=0.1\right)$ as initial data, used the pressure boundary condition with zero data and recorded the time history of the position $x_{1}(t)$ of the spring. Fourier transformation in time gave us the frequency response in Figure 7. We used the same parameter values as above and the angular frequencies correspond to the discrete spectra. We also see the convergence of the numerical spectrum to the continuous one in the sense that the frequencies with high amplitudes in the numerical solution correspond to the eigenfrequencies of the continuous problem.

\subsection{Numerical simulations in the linear case}

The calculations so far has been done with the weak penalty implementation of the boundary and interface conditions. In that case we can prove stability by energy estimates as was done in sections 5.4 and 5.5 above. This is not the most common way to implement coupling or boundary conditions. Often, so called strong implementation (or injection) is used, see [16].

In the injection method, the values of the boundary conditions and coupling condition are directly given to the appropriate variable. For the coupling procedure this means that 


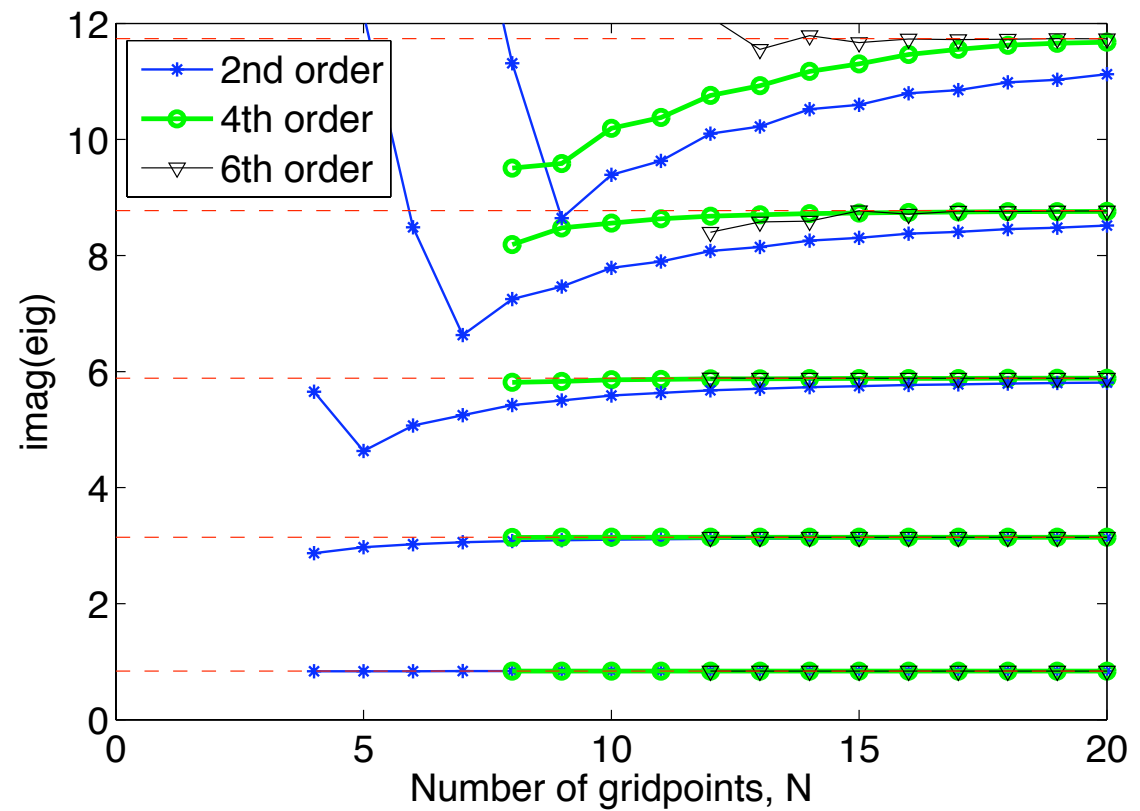

Figure 6: The effect higher order schemes have when computing the five lowest eigenfrequencies. All schemes use weak imposition of boundary conditions.

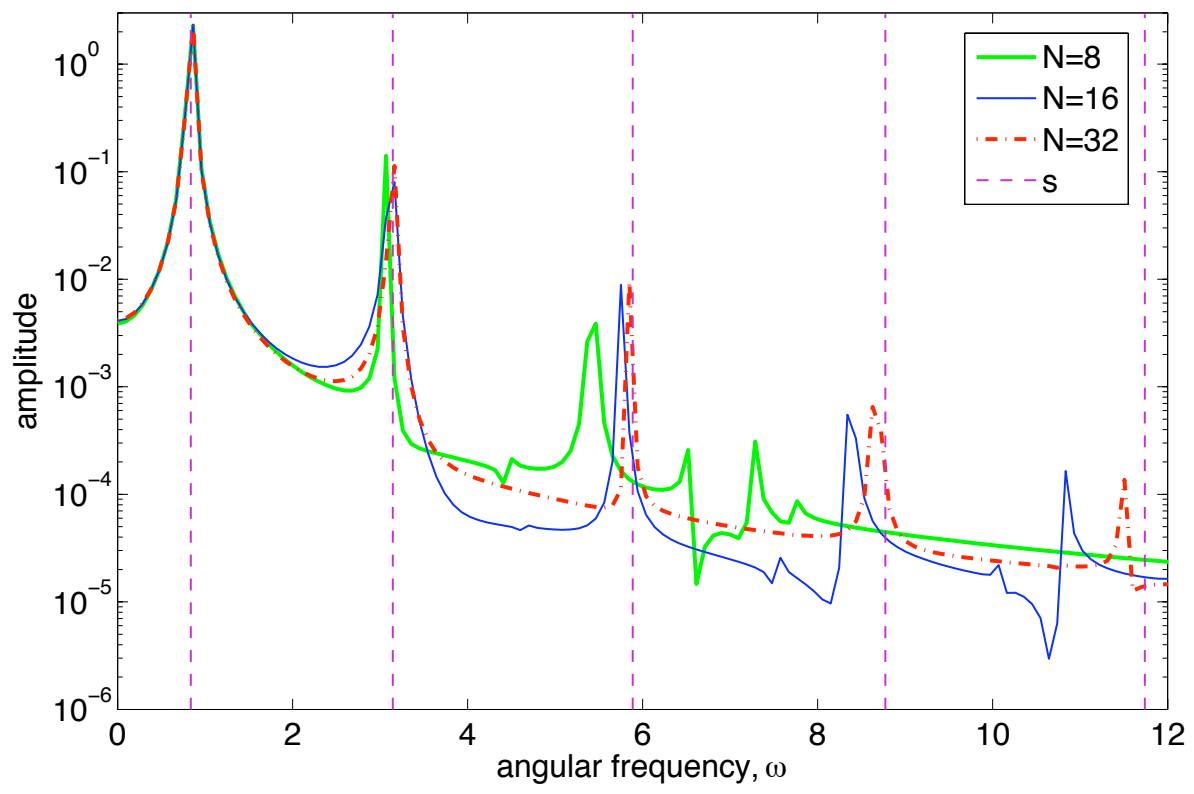

Figure 7: Frequency response for increasing number of grid-points from an initial small displacement of the spring. The continuos frequencies are indicated by dashed lines. 


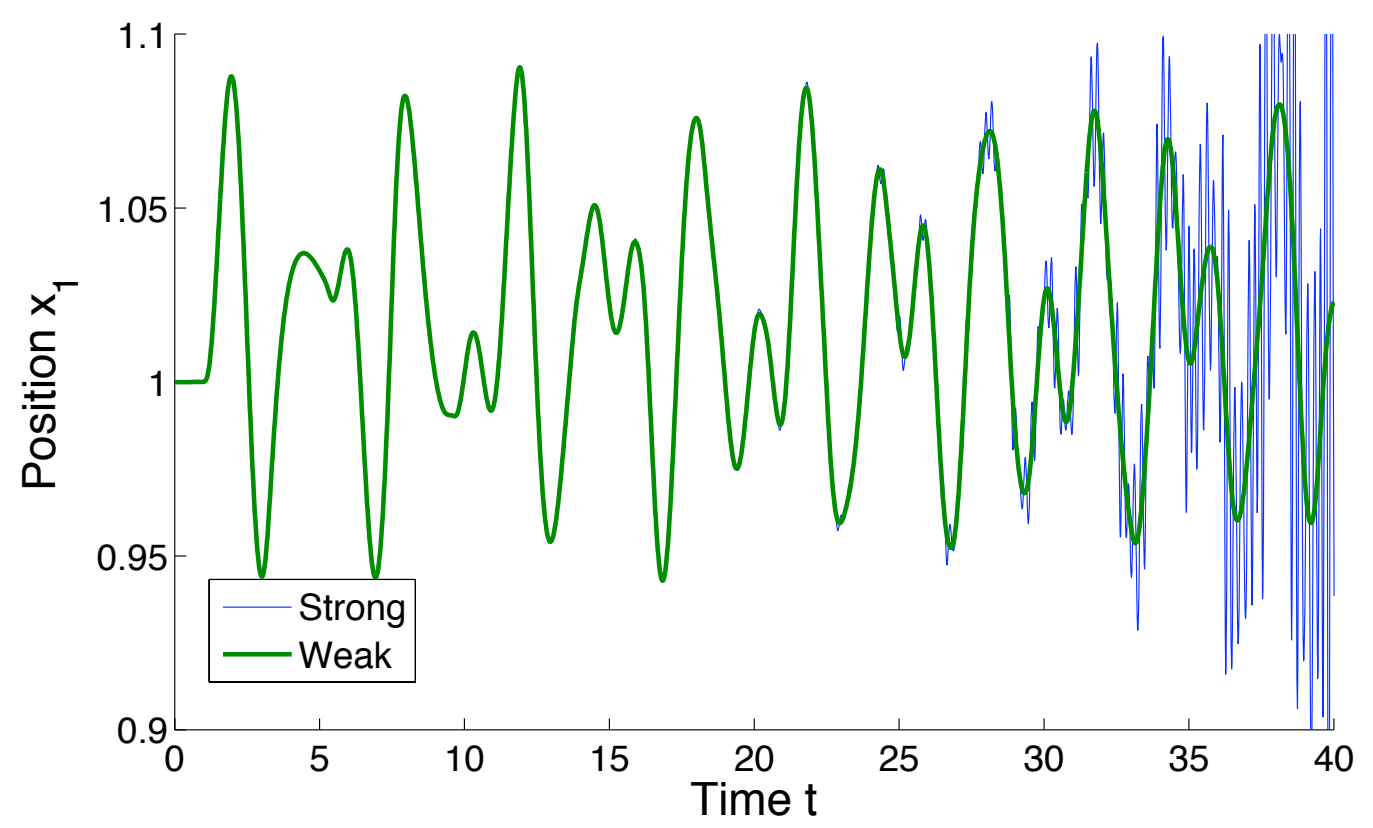

Figure 8: The effect of strong and weak imposition of boundary conditions for $\omega=\pi$. The strong calculation explodes shortly after $t=40$ while the weak calculation remains stable. Note that $\pi$ is not an eigenfrequency.

the value of the spring velocity $V_{2}$ is directly given to the velocity of the flow problem as $u_{N}=V_{2}$. With injection, specific numerical boundary conditions must also be employed (they are pre-computed and integrated in the SBP operator). We have used extrapolation for both the density and temperature as $\rho_{N}=\alpha \rho_{N-1}+\beta \rho_{N-2}$ and $T_{N}=\alpha T_{N-1}+\beta T_{N-2}$. For first order extrapolation we use $\alpha=1, \beta=0$, and for second order $\alpha=2, \beta=-1$.

The effect of the strong boundary procedure along with a stable calculation using the weak form with SBP operators can be seen in Figure 8, where the second order extrapolation has been used. At the left boundary we specify the velocity with an amplitude of 0.1 and the frequency $\omega=\pi$. Note that $\pi$ is not an eigenfrequency. Clearly the weak treatment with SBP operators is superior. The incorrect boundary treatment for the injection method leads to an instability that resembles and can be mistaken for flutter.

Next we study the effect of higher order schemes. High order of accuracy is especially important when dealing with high eigenfrequencies and long time calculations. Figure 9 show that quite different results are obtained using second and fourth order accurate methods. The second order method completely misses the long time growth that is captured by the fourth order accurate method. 


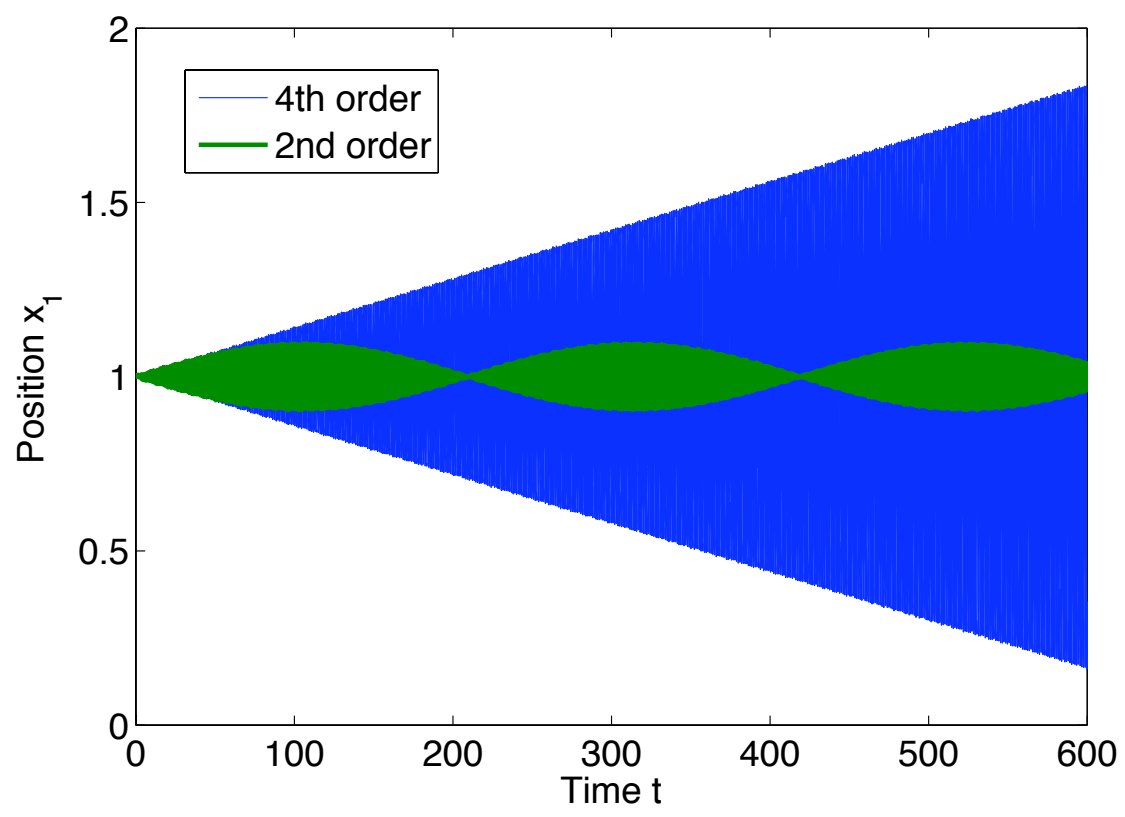

Figure 9: The second order method misses the long time growth of the high frequency mode ( $\omega=$ 5.88659330731852 ) that is captured by the fourth order accurate method.

\subsection{Numerical simulations in the non-linear case}

In this section we return to the difference between the linear and non-linear version of the equations. We start by studying the example shown in Figures 10-12. In these calculations we have used exactly the same boundary and coupling conditions and we are feeding in the pressure at an eigenfrequency of the linear problem at the left boundary. The timestep is the same for the linear and non-linear calculation in each Figure, but varies from figure to figure. In Figures 10-12 we use $\Delta t=2 / 100,2 / 200,2 / 300$ respectively. In space we use $N=64$ grid points. The maximum time-step is well below the stability limit for the 4th order Runge-Kutta scheme applied to the linear problem. We also made sure that the time-step was continuously monitored and kept sufficiently small for the non-linear case, where the spectral radius varies with time.

As expected the linear problem grows linearly in time in all three figures. The nonlinear problem does not. The difference is due to the remaining source term (5.16) which changes sign in an oscillatory manner. For large and medium time-steps, see Figure 1011, the non-linearity does not cause an explosion, instead the growth compared to the linear case is damped. The smallest time-step however, see Figure 12, cause an unlimited growth and a sudden explosion. Note also the higher frequency content in the non-linear calculations.

We investigated this phenomena further and the role of the non-linear term (5.16) is 


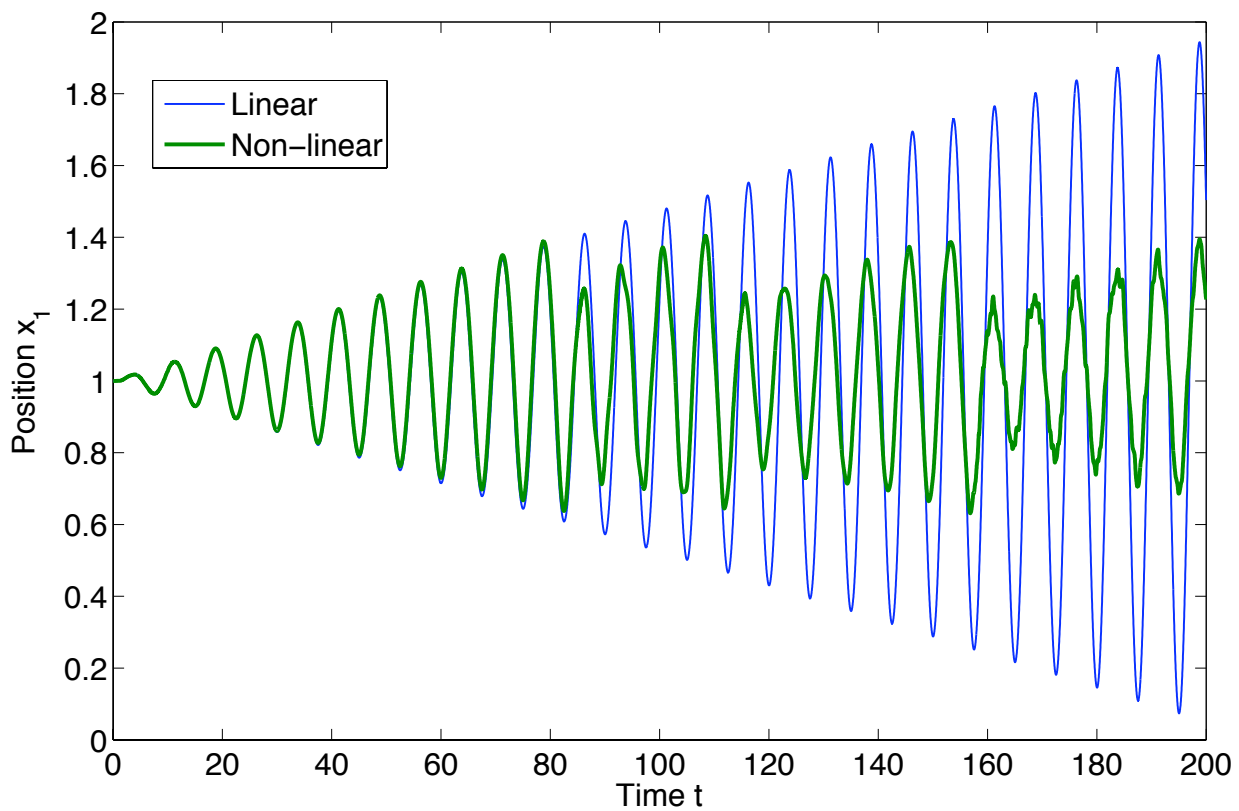

Figure 10: The linear and non-linear response when feeding in pressure variations at the left boundary at an eigenfrequency $(\omega=0.83756479484021)$ of the linear problem. A large time step, $\Delta t=2 / 100$, is used.

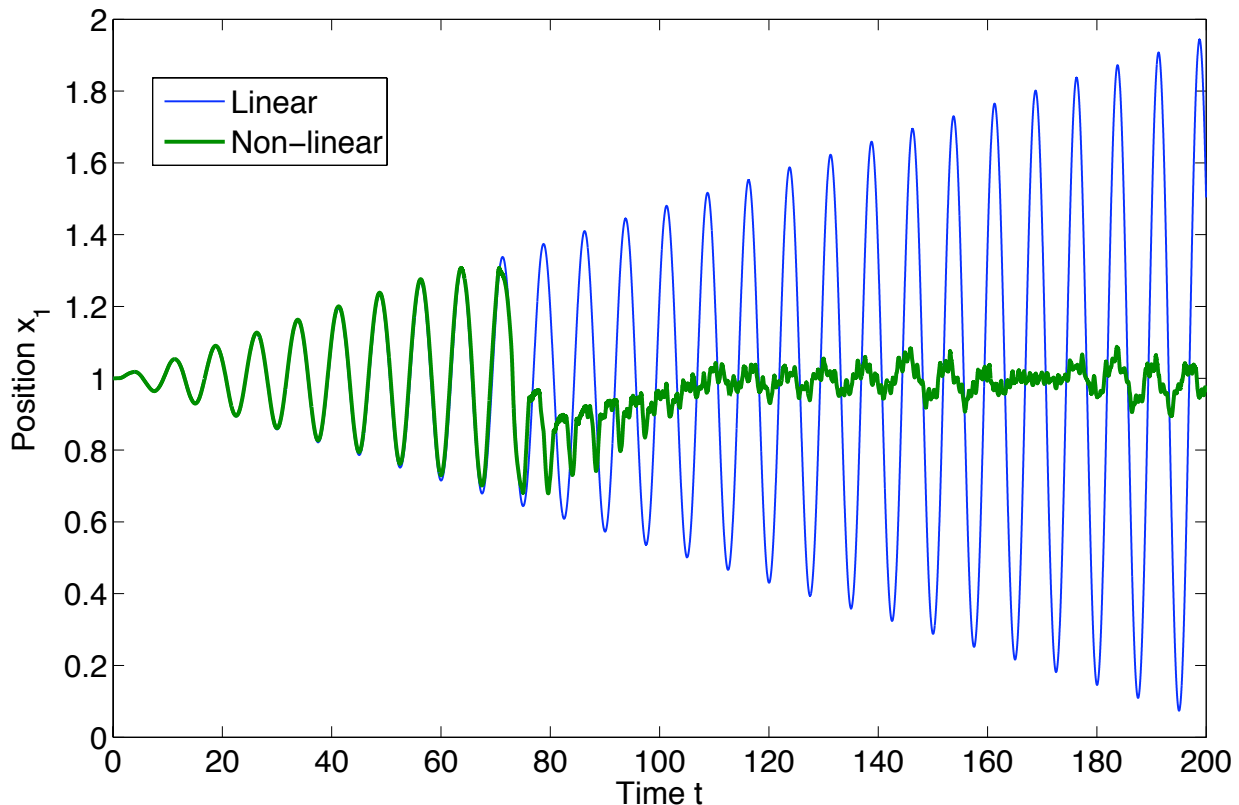

Figure 11: The linear and non-linear response when feeding in pressure variations at the left boundary at an eigenfrequency $(\omega=0.83756479484021)$ of the linear problem. A medium time step, $\Delta t=2 / 200$, is used. 


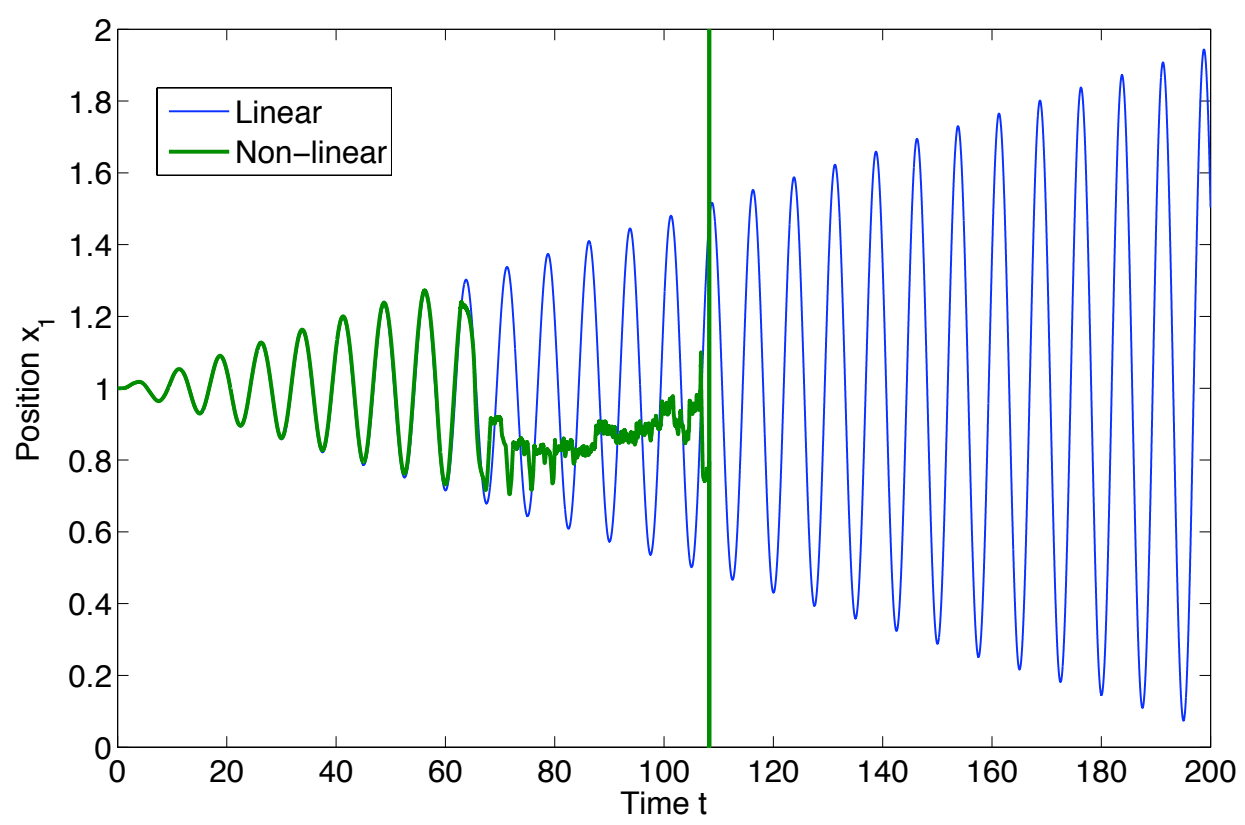

Figure 12: The linear and non-linear response when feeding in pressure variations at the left boundary at an eigenfrequency $(\omega=0.83756479484021)$ of the linear problem. A small time step, $\Delta t=2 / 300$, is used.

clarified in Figure 13 where the eigenvalue with maximum real part is shown as a function of time. The eigenvalues were computed from the matrix in the non-linear equation (5.8) which is formulated more clearly in (A.13). (We are aware of the fact that a nonlinear problem does not have eigenvalues in the same sense as a linear problem and that uniqueness is an issue).

Note that the real part of the non-linear "eigenvalues" become positive and large when $\dot{x}_{1}>0$. Not visible in Figure 13 is the fact that the eigenvalues shift sign as $\dot{x}_{1}$ shifts sign. Also hardly visible is the fact the there are always eigenvalues with positive real part, although small when $\dot{x}_{1}<0$. It is not completely clear why a sufficiently small time step causes an explosion (maybe because the exponential growth can take full advantage of the positive eigenvalues), but it does.

To investigate the unlimited growth in Figure 12 we computed the eigenvalues for that case and the result is shown in Figure 14. The explosion at approximately $t=110 \mathrm{can}$ clearly be correlated with a sudden large growth of the maximum positive real part of the eigenvalues. Figure 14 reveals another interesting aspect. While the position $x_{1}$ has a rather mild behavior with small amplitudes all the way to explosion, the speed of the spring $\dot{x}_{1}$ oscillates wildly already at early times.

As a final investigation of the results in Figures 10-12 we added artificial dissipation of the form

$$
-\theta \Delta x\left(P \otimes I_{3}\right)^{-1} D^{T} D U
$$



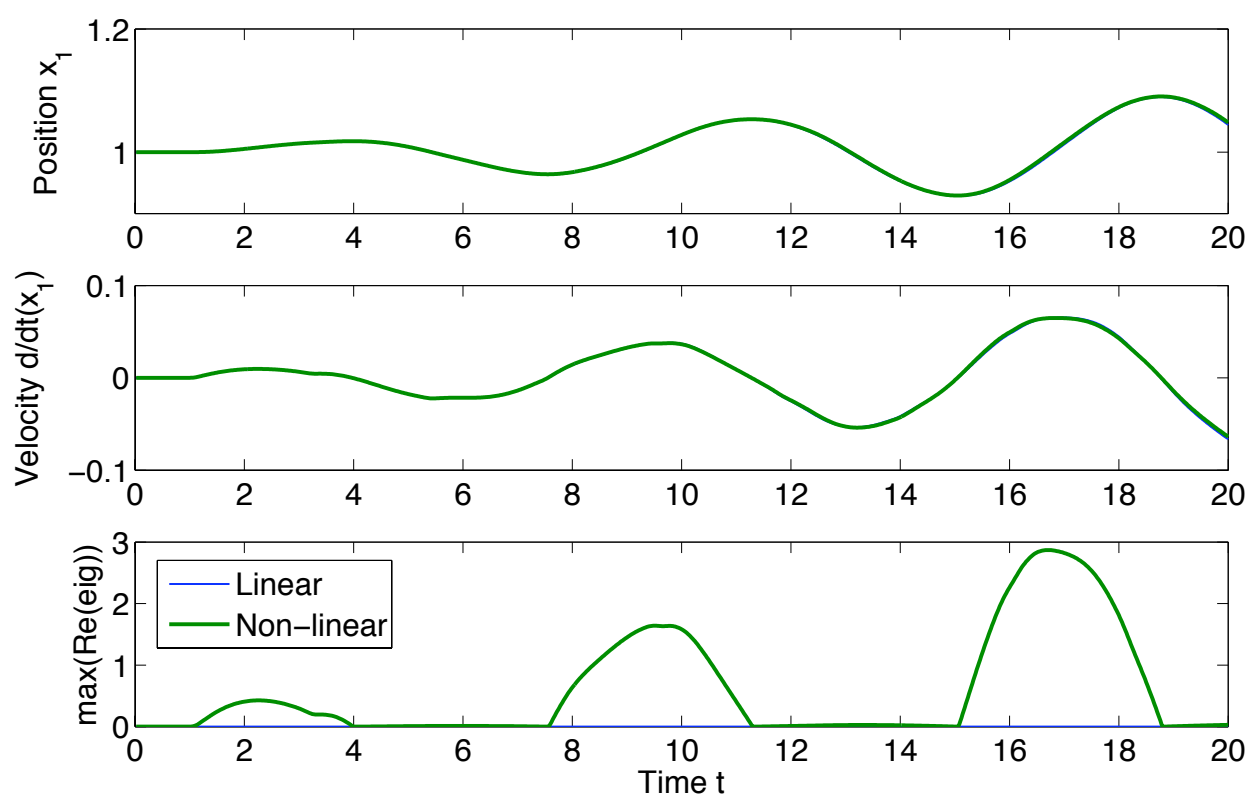

Figure 13: The linear/nonlinear response of pressure variations at the left boundary at an eigenfrequency $(\omega=0.83756479484021)$. The real part of the non-linear "eigenvalues" become positive and large when $\dot{x}_{1}>0$.
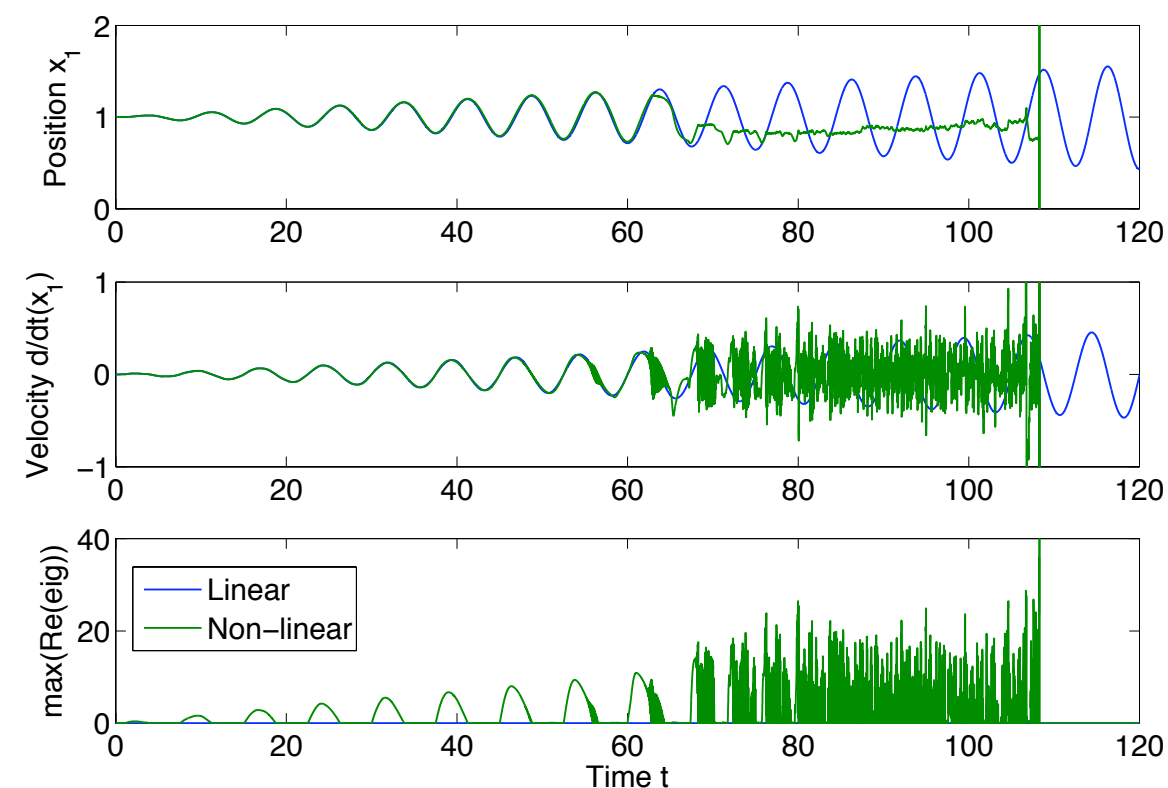

Figure 14: The linear/nonlinear response of pressure variations at the left boundary at an eigenfrequency $(\omega=0.83756479484021)$. The real part of the non-linear "eigenvalues" become very large at "explosion" time. 


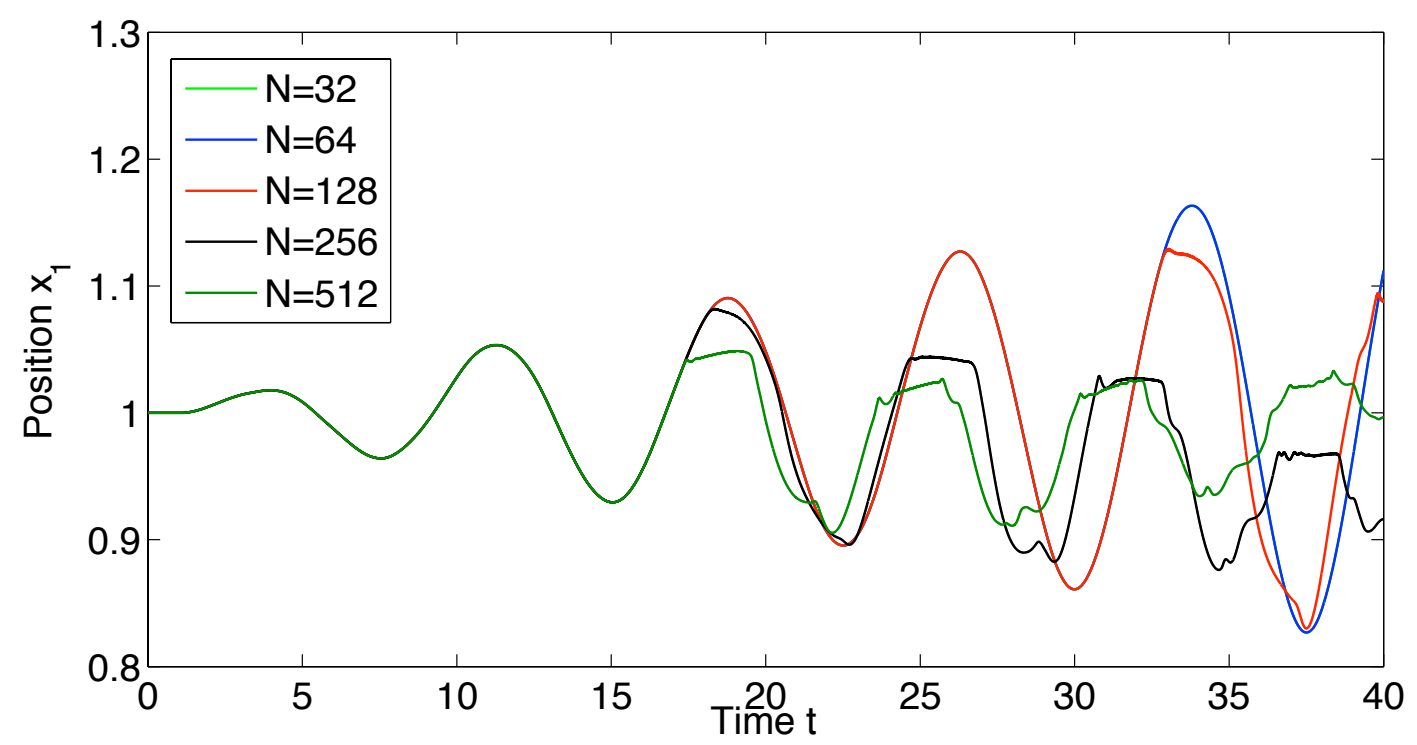

Figure 15: The nonlinear response of pressure variations at the left boundary at an eigenfrequency $(\omega=$ $0.83756479484021)$. Five different grids and $\theta=0$, no artificial dissipation is used.

to the right-hand side of (5.8). The operator $D$ is an undivided difference operator of the appropriate order. The form (6.1) guarantees that accuracy and stability (if it exist) is preserved (see [18] for more details). The results for the final time $t=40$, five different grids $(N=32,64,128,256,512)$, the time-step $\Delta t=4 / 3200$ and four different amounts of artificial dissipation $(\theta=0,1,2,10)$ are shown in Figures 15-18. Note that solutions with many grid-points become non-smooth before solutions with fewer grid-points and that the oscillatory behavior is gradually reduced by increasing the artificial dissipation. For $\theta=10$, most of the oscillations in the solutions are gone. By reducing the time-step even further, we made sure that this phenomenon was not time-step related.

The non-linear explosion in Figure 12 can now be reasonably well understood. The non-linear term in (5.8) generates higher and higher wave-numbers and without dissipation and a stability bound, they can grow and lead to an explosion. Once sufficiently high wave numbers have been generated, no accuracy remains and virtually any result can be obtained (see Figures 10-12). By adding artificial dissipation as in Figures 15-18 we can delay that process. In fact, as long as the solution remains smooth, we have design order of accuracy. However, for a sufficiently fine mesh (and consequently a sufficiently small artificial dissipation term), the potential instability will reappear and might cause an explosion.

Another way of looking at the linear and non-linear problem is shown in Figures 19 and 20 where the effect of a small and large initial displacement is shown. The figures are obtained in the same way as in Figure 7. The spring is given an initial displacement, the pressure boundary condition with zero data is used and the time history of the po- 


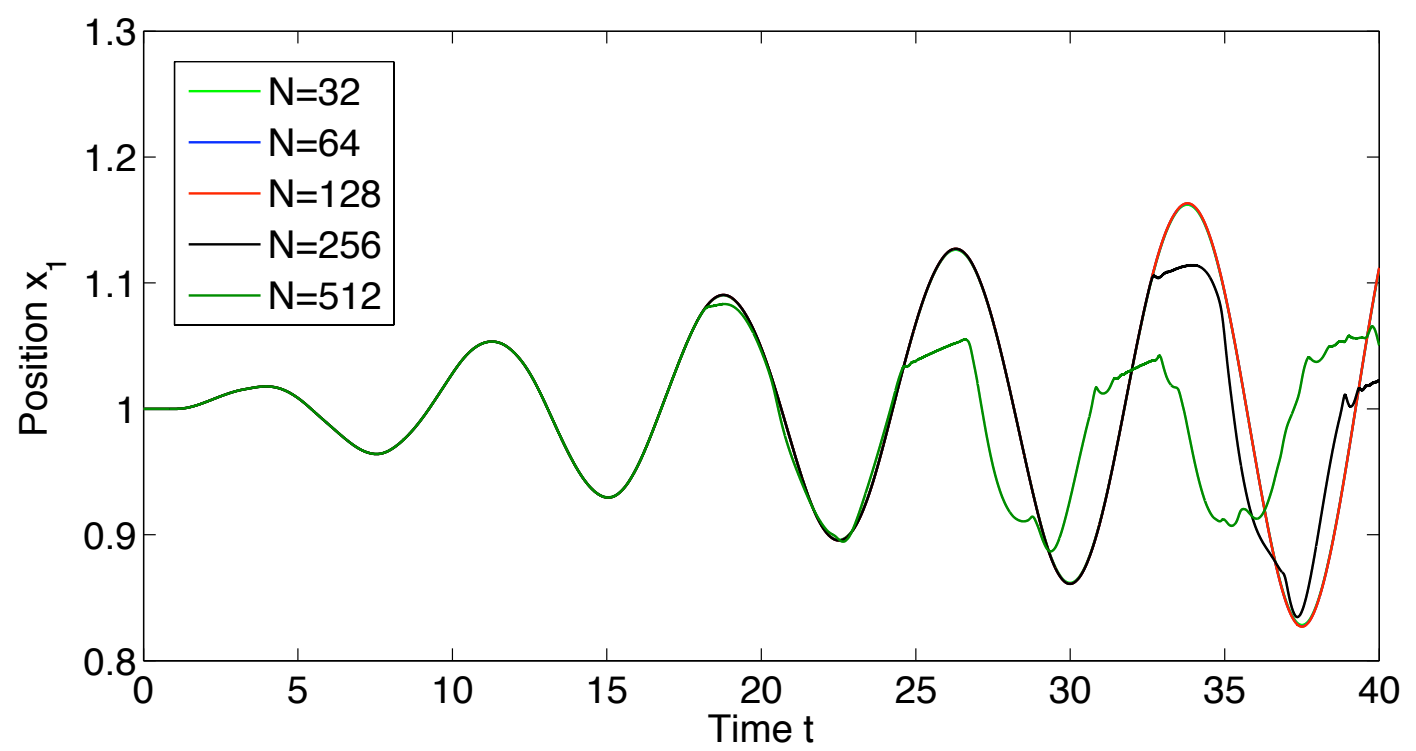

Figure 16: The nonlinear response of pressure variations at the left boundary at an eigenfrequency $(\omega=$ 0.83756479484021 ). Five different grids and $\theta=1$, a small amount of artificial dissipation is used.

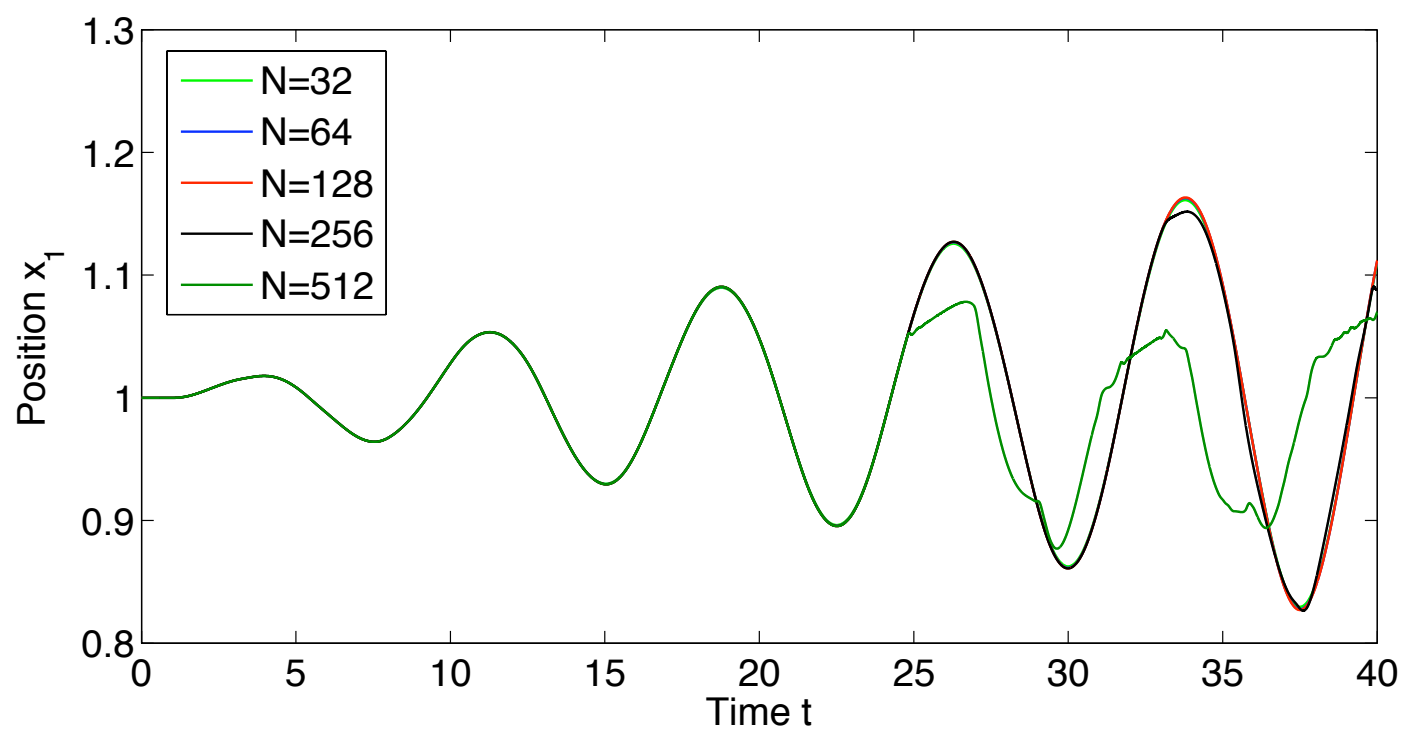

Figure 17: The nonlinear response of pressure variations at the left boundary at an eigenfrequency $(\omega=$ $0.83756479484021)$. Five different grids and $\theta=2$, an intermediate amount of artificial dissipation is used. 


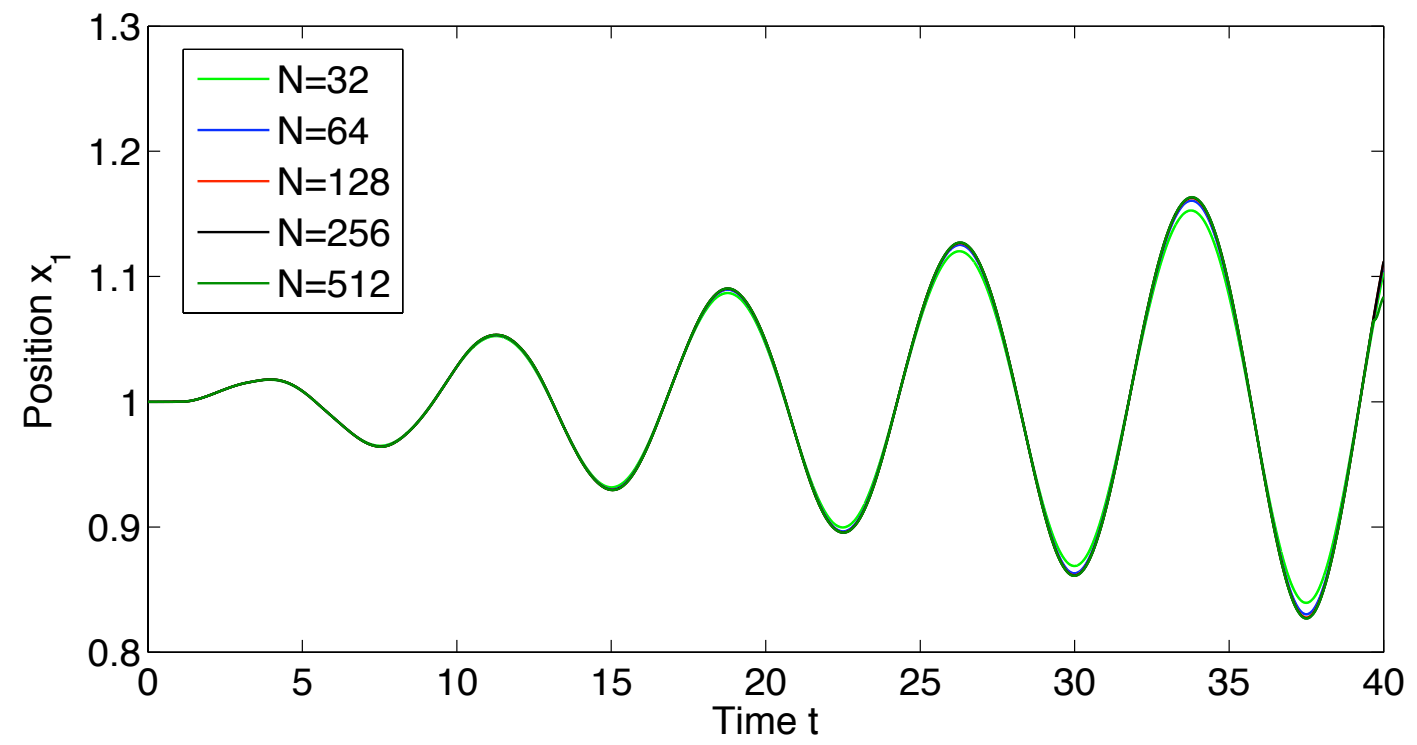

Figure 18: The nonlinear response of pressure variations at the left boundary at an eigenfrequency $(\omega=$ 0.83756479484021 ). Five different grids and $\theta=10$, a large amount of artificial dissipation is used.

sition $1+V_{1}=x_{1}(t)$ of the spring is recorded. Fourier transformation in time give us the frequency response. Clearly, for small amplitudes, the linear theory describes the phenomenon well, while the non-linear effects dominate for large deformations. No artificial dissipation is used in Figures 19 and 20.

Finally we consider the linear and non-linear scheme for a frequency $\omega=2$ which is not an eigenfrequency to the linear problem. We used the pressure boundary condition at the left boundary and increased the amplitude to 0.3. Figure 21 shows the result. The non-linear problem eventually explodes, the linear problem does not. No artificial dissipation is used in Figure 21.

As was shown in section 4 , the non-linear right hand side problem is not well posed in the sense of Definition 2.1 due to the remaining term $V_{2} U^{T} U$ at $\xi=1$ on the right-handside in the energy estimate. The lack of well-posedness results (of course) in a lack of stability as shown in section 5.6. This theoretical deficiency does not necessarily lead to an unbounded growth which was exemplified in Figures 10-12 and 19-20.

One possibility to correct the lack of well-posedness would be to add a boundary condition at the right boundary when $V_{2}$ is positive. However, it is difficult to find a (non-awkward) boundary condition that removes the growth term. Also, even if that could be done, the data for the boundary condition do not really exist for the Euler case. Another possibility would be to change equation set and use the Navier-Stokes equations which naturally allows for more boundary conditions and types of data.

Our conclusion is that the lack of well-posedness cannot be corrected in a straight forward manner for our problem. The lack of well-posedness and stability for this model 


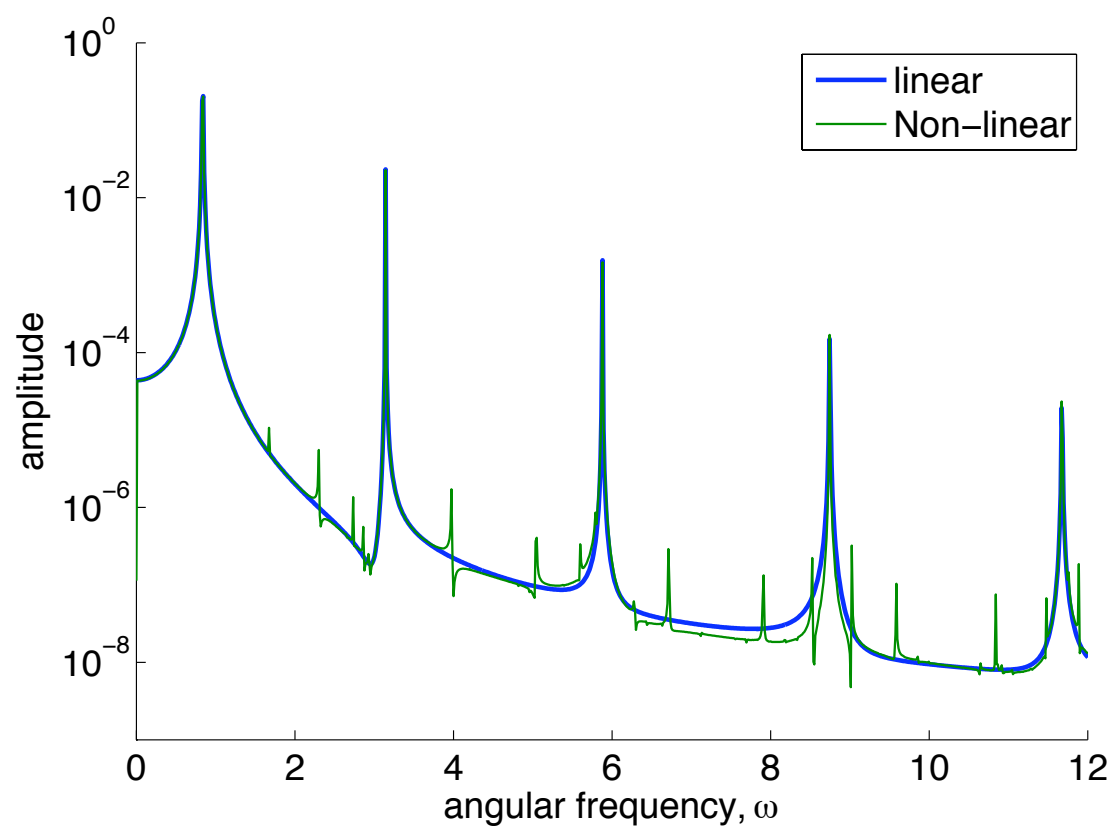

Figure 19: The frequency response for a small initial displacement, $\Delta x=0.01$.

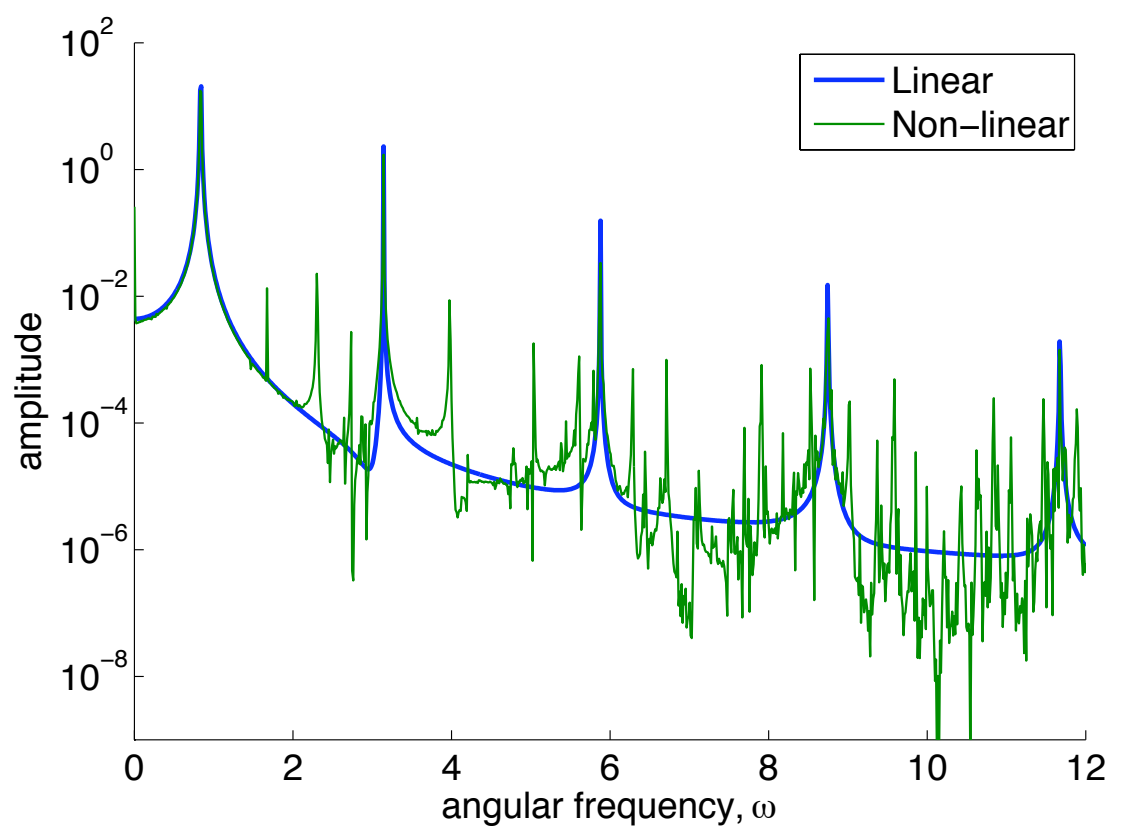

Figure 20: The frequency response for a large initial displacement, $\Delta x=0.10$. 

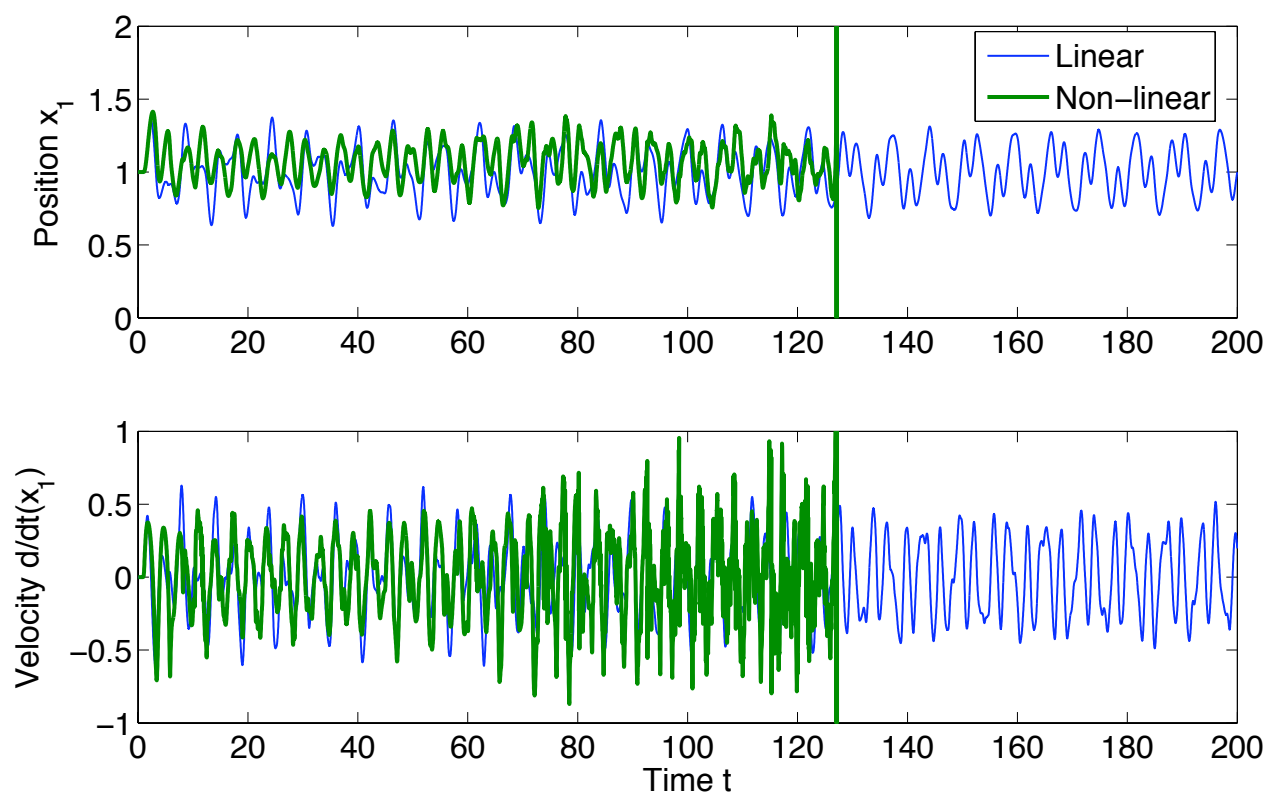

Figure 21: The linear and non-linear response for a pressure variation at the left boundary at $\omega=2$ and the amplitude $=0.3$. $\omega=2$ is not an eigenfrequency. The non-linear problem explodes, the linear problem does not.

problem might exist even for three-dimensional FSI calculations using the Euler equations.

\section{Summary and conclusions}

We have investigated well-posedness of FSI problems. As a flow model we have used the linearized Euler equations in one dimension and as the structure model, a spring. We have studied both the non-linear and linear problem. The non-linearity stems from large deflections and high speed of the spring.

It was shown that the generally accepted formulation of the linear FSI problem is the only possible one. It was also shown that coupling in the linear case can be derived using the demand for well-posedness as the main tool. The non-linear version of the problem is not well-posed in the classical sense.

For the linear problem we derived and proved that the numerical coupling using summation-by-parts operators and weak boundary conditions is stable. We verified our numerical procedure by using the method of manufactured solutions and by showing that the numerical eigenfrequencies converge to the analytical eigenfrequencies (the spectrum) of the coupled partial differential equations.

We compared our numerical procedure with the common way of using injection to impose the boundary and coupling conditions for the linear problem. Our procedure is 
stable and gave correct results while the injection procedure can lead to instabilities that easily can be interpreted as flutter. The effect of higher order schemes was studied. It was shown that the higher order accurate approximations converges faster and more easily capture the effects related to the higher eigenfrequencies.

Non-linear calculations using exactly the same coupling procedures as in the linear case were performed. It was found that the non-linear and linear calculations agree well for small deflections, which verified the non-linear scheme also, since the linear scheme is provable correct. It was also shown that for large deflections and high speed of the spring, clear differences exist.

The lack of well-posedness (and stability) for the non-linear problem did not lead to an unbounded growth for all the cases we studied. In fact, the growth in the non-linear problem could be modified in various ways, for example by the choice of time step. For large time steps (although within the linear stability limit), no growth was observed. On the other hand, for sufficiently small time steps, unbounded growth was obtained.

It was concluded that higher and higher wave-numbers were generated in the nonlinear problem. Without dissipation and a stability bound, they can grow and lead to explosions. Once sufficiently high wave numbers have been generated, no accuracy remains and virtually any result can be obtained. By adding artificial dissipation, that process can be modified and delayed but not removed.

Finally, the comparison between the linear and non-linear problem was discussed and it was concluded that it would be difficult to correct the lack of well-posedness for this problem. It was also noted that the lack of well-posedness and stability for this model problem might very well exist even for realistic three-dimensional FSI calculations using the Euler equations.

\section{Acknowledgments}

We thank Dr. Arnaud Malan at the Computational Aerodynamics Branch, Division of Defense, Peace, Safety and Security, CSIR South Africa for the support for this work.

\section{A The time integration procedure}

We start by constructing the scheme for the linear problem. The non-linear scheme is easily obtained by a few small modifications. 


\section{A.1 The linear scheme}

We will integrate the coupled system as one large system of ordinary differential equations. The formulation including boundary conditions is

$$
\begin{aligned}
U_{t} & =-\left(P^{-1} Q \otimes A\right) U+\left(P^{-1} E_{0} \otimes \Pi\right)\left(U-G_{0}\right)+\left(P^{-1} E_{N} \otimes \Sigma\right)\left(U-G_{N}\right) \\
V_{t} & =-\tilde{B} V+\tilde{F}(U),
\end{aligned}
$$

where

$$
\tilde{B}=M^{-1} K=\left[\begin{array}{cc}
0 & -1 \\
k / m & b / m
\end{array}\right], \quad \tilde{F}=M^{-1} F=\left[\begin{array}{c}
0 \\
p_{N} / m
\end{array}\right]
$$

By introducing the notation $\tilde{A}=\left(P^{-1} Q \otimes A\right), \tilde{\Sigma}=\left(P^{-1} Q \otimes \Sigma\right)$ and $\tilde{\Pi}=\left(P^{-1} Q \otimes \Pi\right)$ we can rewrite the system as

$$
\begin{aligned}
U_{t} & =(-\tilde{A}+\tilde{\Sigma}+\tilde{\Pi}) U-\tilde{\Pi} G_{0}-\tilde{\Sigma} G_{N} \\
V_{t} & =-\tilde{B} V+\tilde{F}
\end{aligned}
$$

where the coupling between (A.4) and (A.5) is included in $\tilde{\Sigma} G_{N}$ and $\tilde{F}(U)$ as

$$
\begin{aligned}
& \tilde{\Sigma} G_{N}=P_{N N}^{-1} \bar{\rho} \bar{c}^{2} V_{2}[0, \cdots 0,1 / \sqrt{\gamma} 0 \sqrt{(\gamma-1) / \gamma}]^{T} \\
& \tilde{F}(U)=\left[0,\left(U_{3 N+1} / \sqrt{\gamma}+U_{3 N+3} \sqrt{(\gamma-1) / \gamma}\right) / m\right]^{T} .
\end{aligned}
$$

Next we form $y=[U ; V]$ as a vector of length $3(N+1)+2$ where $N+1$ is the number of grid-points. This gives us the final form of the system which is now ready for time integration.

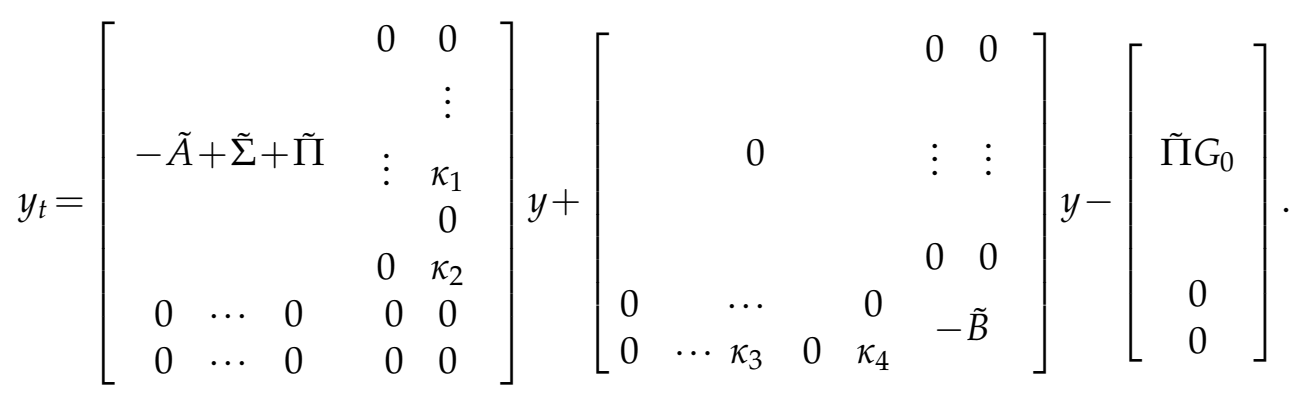

The coupling terms in (A.8) are

$$
\kappa_{1}=-\frac{P_{N N}^{-1} \bar{\rho} \bar{c}^{2}}{\sqrt{\gamma}}, \quad \kappa_{2}=-\frac{P_{N N}^{-1} \bar{\rho} \bar{c}^{2} \sqrt{(\gamma-1)}}{\sqrt{\gamma}}, \quad \kappa_{3}=\frac{1}{m \sqrt{\gamma}}, \quad \kappa_{4}=\frac{\sqrt{(\gamma-1)}}{m \sqrt{\gamma}} .
$$




\section{A.2 The non-linear scheme}

The discrete version of the non-linear flow equation is written as

$$
\left(1+V_{1}\right) U_{t}=-\tilde{A} U+V_{2} \tilde{C} U+\tilde{\Pi}\left(U-G_{0}\right)+\tilde{\Sigma}\left(U-G_{N}\right)
$$

where $\tilde{A}, \tilde{\Sigma}$ and $\tilde{\Pi}$ are defined above and $\tilde{C}=\left(\Xi P^{-1} Q+P^{-1} Q \Xi-I_{N}\right) \otimes I_{3} / 2$. The corresponding system to (A.4) and (A.5) in the nonlinear case becomes

$$
\begin{aligned}
\left(1+V_{1}\right) U_{t} & =\left(-\tilde{A}+V_{2} \tilde{C}+\tilde{\Sigma}+\tilde{\Pi}\right) U-\tilde{\Pi} G_{0}-\tilde{\Sigma} G_{N} \\
V_{t} & =-\tilde{B} V+\tilde{F} .
\end{aligned}
$$

The definitions of all the terms in (A.11) and (A.12) can be found in section A.1 above. The non-linear coupled equations formulated as a single system corresponding to (A.8) is

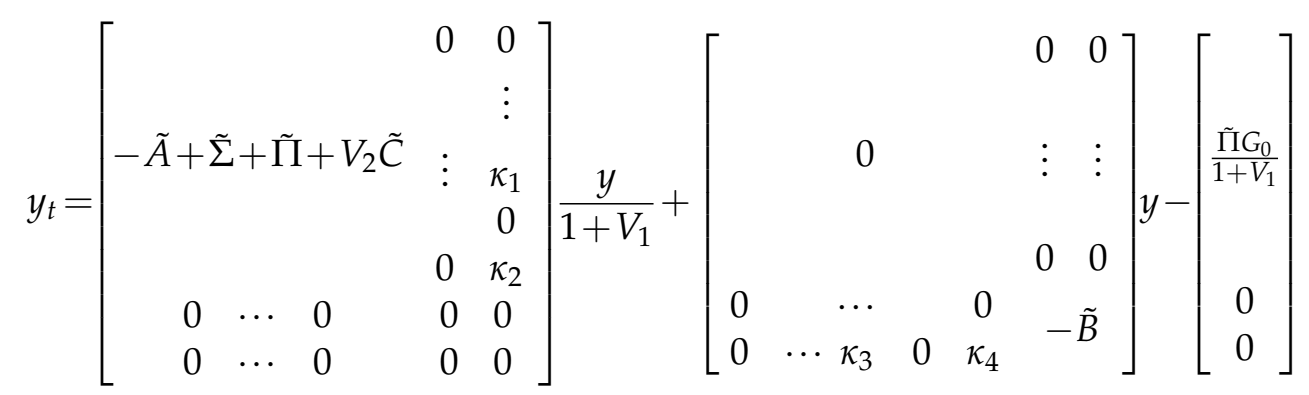

where the coupling terms in (A.13) are the same as the ones in (A.8) and given by (A.9).

\section{References}

[1] S. Abarbanel and D. Gottlieb. Optimal time splitting for two- and three-dimensional NavierStokes equations with mixed derivatives. Journal of Computational Physics, 41:1-43, 1981.

[2] J. Alonso and A. Jameson. Fully-implicit time-marching aeroelastic solutions. In 32th Aerospace Sciences Meeting, number AIAA Paper 94-0056, Reno, January 1994.

[3] K.J. Bathe, C. Nitikitpaiboon, and X. Wang. A mixed discplacement-based finite element formulation for acoustic fluid-structure interaction. Computers and Structures, 56(2-3):225237, 1995.

[4] P. Geuzaine C. Farhat and G. Brown. Application of a three-field nonlinear fluid-structure formulation to the prediction of the aeroelastic parameters of an $\mathrm{f}-16$ fighter. Computers and Fluids, 32:3-29, 2003.

[5] M. H. Carpenter, J. Nordström, and D. Gottlieb. Revisiting and extending interface penalties for multidomain summation-by-parts operators. Journal of Scientific Computing, 2009.

[6] M.H. Carpenter, J. Nordström, and D. Gottlieb. A stable and conservative interface treatment of arbitrary spatial accuracy. Journal of Computational Physics, 148:341-36, 1999.

[7] M. Dalenbring and A. Zdunek. On the use of three-dimensional h- and p-version finite elements in solving vibration response problems. Journal of Sound and Vibration, 288(4-5):907$929,2005$. 
[8] E.H. Dowell and D. Tang. Nonlinear aeroelasticity and unsteady aerodynamics. AIAA Journal, 40(9):1697-1707, September 2002.

[9] J.F. Gerbeau and M. Vidrascu. A quasi-newton algorithm based on a reduced model for fluid-structure interaction problems in blood flows. Mathematical Modelling and Numerical Analysis, 37(4):631-647, 2003.

[10] C.J. Greenshields and H.G. Weller. A unified formulation for continuum mechanics applied to fluid-structure interaction in flexible tubes. International Journal for Numerical Methods in Engineering, 64:1575-1593, 2005.

[11] Kreiss H.-O. Gustafsson, B. and Oliger. Time-dependent problems and difference methods. J. John Wiley and Sons, 1995.

[12] J. De Hart, GWM Peters, PJG Schreurs, and FPT Baaijens. A three-dimensional computational analysis of fluid-structure interaction in the aortic valve. Journal of Biomechanics, 36(1):103-112, 2003.

[13] H.-O. Kreiss and G. Scherer. Finite element and finite difference methods for hyperbolic partial differential equations, in: C. De Boor (Ed.), Mathematical Aspects of Finite Elements in Partial Differential Equation. Academic Press, New York, 1974.

[14] V.Milisic M.A.Fernandez and A.Quateroni. Analysis of a geometrical multiscale blood flow model based on the coupling of ODE's and hyperbolic PDE's. Multiscale Modeling and Simulation, 4:215-236, 2005.

[15] E. Di Martino, G. Guadagni, A. Funaro, and G. Ballerini. Fluid-structure interaction within realistic three-dimensional models of the aneurysmatic aorta as a guidance to asess the risk of rupture of the aneurysm. Medical Engineering and Physics, 23(9):647-655, 2001.

[16] K. Mattsson. Boundary procedures for summation-by-parts operators. Journal of Scientific Computing, 18(1):133-153, 2003.

[17] K. Mattsson and J. Nordström. Summation by parts operators for finite difference approximations of second derivatives. Journal of Computational Physics, 199:503-540, 2004.

[18] K. Mattsson, M. Svärd, and J. Nordström. Stable and accurate artificial dissipation. Journal of Scientific Computing, 21(1):57-79, 2004.

[19] J. Nordström. The use of characteristic boundary conditions for the Navier-Stokes equations. Computers \& Fluids, 24(5), 1995.

[20] J. Nordström. Conservative finite difference approximations, variable coefficients, energy estimates and artificial dissipation. Journal of Scientific Computing, 29:375-404, 2006.

[21] J. Nordström and M. H. Carpenter. Boundary and interface conditions for high order finite difference methods applied to the Euler and Navier-Stokes equations. Journal of Computational Physics, 148:621-645, 1999.

[22] J. Nordström and M. H. Carpenter. High-order finite difference methods, multidimensional linear problems and curvilinear coordinates. Journal of Computational Physics, 173:149-174, 2001.

[23] B. Strand. Summation by parts for finite difference approximation for $d / d x$. Journal of Computational Physic, 110(1):47-67, 1994.

[24] X. Wang and K.J. Bathe. Displacement/pressure based mixed finite element formulations for acoustic fluid-structure interaction problems. International Journal for Numerical Methods in Engineering, 40(11):2001-2017, 1998. 